\title{
Periodic Overlayers and Moiré Patterns: theoretical studies of geometric properties
}

\begin{abstract}
Klaus Hermann
Fritz-Haber-Institut der Max-Planck-Gesellschaft, Faradayweg 4-6, D-14195 Berlin, Germany; email: hermann@fhi-berlin.mpg.de
\end{abstract}

\begin{abstract}
Single crystal surfaces with periodic overlayers, such as graphene on hexagonal metal substrate, are found to exhibit, apart from their intrinsic periodicity, additional long-range order expressed by approximate surface lattices with large lattice constants. This phenomenon can be described geometrically analogous to lateral interference effects resulting in periodic moiré patterns which are characterized by 2-dimensional moiré lattices. Here we discuss in detail the mathematical formalism determining such moiré patterns based on concepts of 2-dimensional Fourier transformation including coincidence lattices. The formalism provides simple relations that allow to calculate possible moiré lattice vectors in their depencence on rotation angles $\alpha$ and and scaling factors $\mathrm{p}_{1}, \mathrm{p}_{2}$ for periodic $\left(\mathrm{p}_{1} \times \mathrm{p}_{2}\right) \mathrm{R} \alpha$ overlayers on substrate surfaces described by general Bravais lattices. Specific emphasis will be put on hexagonal lattices where experimental data are available.
\end{abstract}




\section{INTRODUCTION}

Surfaces of regular single crystals can exhibit a multitude of structures which deviate from those of simple bulk termination [1,2], reflecting the changed atom environment and composition at the surface. These structural deviations have also been found for adsorbed overlayers of foreign atoms or molecules on single crystal substrates [3] where the overlayers and the topmost substrate monolayers affect each other geometrically. Here surfaces with periodic overlayers, whose 2dimensional periodicity can be derived from that of the underlying substrate monolayer by rotation about the surface normal and by scaling of corresponding lattice constants, are of particular interest. These surfaces are usually denoted as $\left(\mathrm{p}_{1} \times \mathrm{p}_{2}\right) \mathrm{R} \alpha$ structured using the Wood notation $[4,5]$ where $\alpha$ refers to the rotation angle of the overlayer lattice vectors relative to those of the underlying substrate monolayer and $\mathrm{p}_{1}, \mathrm{p}_{2}$ are scaling factors connecting lattice constants of the overlayer with those of the substrate. This includes overlayers of foreign atoms or molecules on substate as well as clean surfaces with the topmost overlayer reconstructed which can be treated at the same footing.

Surfaces with $\left(\mathrm{p}_{1} \times \mathrm{p}_{2}\right) \mathrm{R} \alpha$ overlayer lattices are found to exhibit, apart from their intrinsic periodicity and depending on the overlayer-substrate coupling, additional long-range order with an approximate periodicity expressed by surface lattices with rather large lattice constants. This is manifested by 2-dimensional quasi-periodic variations of atom distances between the overlayer and the substrate (topographic buckling) which includes smaller lateral atom shifts (local lateral surface distortion). The geometric variations can be observed for example by surface X-ray diffraction (SXRD) and result in periodic changes of the electronic structure which can be probed by surface tunneling microscopy (STM). An example is graphene on $\mathrm{Ru}(0001)$ substrate [6-12] where experimental SXRD studies [6] suggest a commensurate phase with a coincidence lattice described by a supercell of $(25 \times 25)$ carbon honeycombs on a $(23 \times 23)$ supercell of the hexagonal $\mathrm{Ru}(0001)$ substrate. This was simulated by DFT calculations with different (smaller than observed) coincidence lattices [7, 9-12] where Fig. 1 shows results for the (13x13) C on (12x12) $\mathrm{Ru}(0001)$ [7] case reflecting an overlayer lattice scaled isotropically by $\mathrm{p}_{1}=\mathrm{p}_{2}=12 / 13$ with respect to that of the Ru substrate without rotation, $\alpha=0^{\circ}$. Other examples include graphene monolayers on graphene [13-16] where the origin of the long-range topographic modulation was argued to be due to overlayer rotation [16]. Further, quasi-periodic hexagonal patterns have been observed for graphene on $\mathrm{Rh}(111)$ $[12,17], \operatorname{Ir}(111)[18,19]$, and $\operatorname{Pt}(111)[17,20]$, on $\mathrm{Cu}(111)$ [21], as well as for hexagonal boron nitride, h-BN, layers on $\operatorname{Ru}(0001)$ [12, 22], $\operatorname{Pd}(111)$ [23], and $\mathrm{Rh}(111)$ [24, 25]. Finally, rotated hex- 
agonal $\mathrm{Ag}$ overlayers on $\mathrm{Ni}(111)$ substrate have been discussed in the literature [26, 27]. Here the influence of both overlayer rotation and differences in the lattice constants of the overlayer and the substrate have been considered to explain corresponding large supercell periodicities.

Obviously, the existence of additional quasi-periodic long-range order in these surface systems results from a balance between the electronic overlayer - substrate coupling, often rather weak, and binding within the overlayer which may both be quite difficult to evaluate in their local details. However, the basic origin of the quasi-periodicity can be described by considering the geometric interplay of the overlayer lattice superimposed with that of the substrate surface. For a normal view to the surface, this can give the visual impression of 2-dimensional spatial interference as exemplified in Fig. 1a for the graphene on $\mathrm{Ru}(0001)$ system. The effect has been discussed in terms of 2dimensional periodic moiré patterns, well known and often unwanted in digital image processing [28]. Thus, the truly 2-dimensional concept of moiré patterns and moiré lattices as a result of overlaying rigid periodic arrays of objects is adapted to describe geometric aspects of 3-dimensional surface regions. Clearly, both the overlayer and the underlying substrate will be strained locally leading to quasi-periodic overlayer buckling and local lateral distortions in addition to lattice rotation and scaling. However, in an approximate treatment, neglecting minor local distortions, the basic properties of the additional geometric surface modulation may be associated with moiré pattern formation. Here the periodic patterns consisting of local surface regions (moiré "spots", termed moirons in the following) are interpreted as consequences of geometric interference of the periodic overlayer and the substrate monolayer.

The geometric structure of local surface regions forming moirons can be quite complex in detail. In particular, the periodicity of moirons suggested visually by corresponding moiré lattices for a given overlayer rotation or scaling can only be approximate. Moirons separated by moiré lattice vectors may differ somewhat in their geometric detail yielding a quasi-periodic arrangement rather than strict periodocity. Only if the surface system can be described by a coincidence lattice the moiron arrangement is truly periodic, however, with its periodicity determined by the coincidence lattice rather than the moiré lattice. Further, the actual size and shape of moirons depends strongly on the rotation and scaling of the overlayer lattice relative to that of the substrate and ranges from almost circular compact spots to spirals or stripes as will be shown below. In realistic surface systems moiré patterns become evident by topographic buckling, see Fig. 1, combined with corresponding electronic properties. The present theoretical treatment simulates corresponding monolay- 
ers by periodic arrangements of balls in two dimensions and the moiré interference effect becomes visible by the laterally varying transparency in a perpendicular view. Here areas of high transparency of the superimposed overlayer and substrate monolayer are considered to model moirons at the surface.

The formal description of fictitious lattices resulting from moiré patterns can be achieved by 2dimensional Fourier theory including coincidence lattices. This will be examined in detail in the present study yielding analytical formulas for periodicity vectors of the lattices approximating possible quasi-periodic moiré patterns at surfaces with $\left(\mathrm{p}_{1} \times \mathrm{p}_{2}\right) \mathrm{R} \alpha$ overlayers. While some geometric aspects of moiré patterns have been discussed earlier for hexagonal overlayers [18-20] the present theoretical treatment provides a unified formalism valid for general periodic overlayers on substrate of any Bravais lattice type.

Section 2 introduces the general formalism to describe moiré interference patterns which can appear at surfaces of a given Bravais lattice type. Section 3 discusses results from the general formalism for different geometric scenarios, isotropically scaled (p x p), rotated (1x1) R $\alpha$, rotated and isotropically scaled ( $\mathrm{p}$ x p) R $\alpha$, and general $\left(\mathrm{p}_{1} \times \mathrm{p}_{2}\right) \mathrm{R} \alpha$ overlayers, see Secs. 3.1 to 3.4. Here hexagonal surface structures, which have been examined by experiment, are specifically emphasized. Finally, in Section 4 the most important results from the theoretical treatment are summarized.

\section{THEORETICAL DETAILS}

In this section we discuss basic details of the general formalism which can be used to describe quasi-periodic moiré interference patterns at surfaces of a given Bravais lattice type. The resulting (approximate) moiré lattice is defined by 2-dimensional lattice vectors $\underline{R}_{M 1}, \underline{R}_{M 2}$ which can be obtained in analytic form applying 2-dimensional Fourier theory. This will be shown in Sec. 2.1 for most general overlayers using (2x2) matrix notation [5] while Sec. 2.2 considers moiré lattices for overlayers described as $\left(\mathrm{p}_{1} \times \mathrm{p}_{2}\right) \mathrm{R} \alpha$ in Wood notation [4, 5]. Further, Sec. 2.3 discusses moiré patterns for surfaces forming coincidence lattices.

\subsection{General formalism}


As a starting point we consider a function $\mathrm{f}_{\mathrm{S}}(\mathrm{r})$, describing spatial properties of a substrate surface which is periodic in two dimensions with its periodicity defined by lattice vectors $\underline{R}_{01}, \underline{R}_{02}$. Thus, function $\mathrm{f}_{\mathrm{S}}(\underline{\mathrm{r}})$ can be represented by an infinite Fourier series as

$$
\mathrm{f}_{\mathrm{S}}(\underline{\mathrm{r}})=\sum_{\mathrm{j}, \mathrm{k}} \mathrm{c}_{\mathrm{j}, \mathrm{k}}^{\mathrm{S}} \exp \left(\mathrm{i}\left[\mathrm{j} \underline{\mathrm{G}}_{\mathrm{o} 1}+\mathrm{k} \underline{\mathrm{G}}_{\mathrm{o} 2}\right] \underline{\mathrm{r}}\right), \mathrm{j}, \mathrm{k} \text { integer }
$$

with, in general, complex valued coefficients $c_{j, k}^{S}$ and reciprocal lattice vectors $\underline{G}_{01}, \underline{G}_{02}$ which can be derived from lattice vectors $\underline{\mathrm{R}}_{\mathrm{o} 1}, \underline{\mathrm{R}}_{\mathrm{o} 2}$ by corresponding orthogonality relations [5]. Similarly, functions $\mathrm{f}_{\mathrm{O}}(\underline{\mathrm{r}})$, describing spatial properties of a periodic overlayer, characterized in its periodicity by lattice vectors $\underline{\mathrm{R}}_{01}, \underline{\mathrm{R}}_{\mathrm{o} 2}$, can be represented by an infinite Fourier series as

$$
\mathrm{f}_{\mathrm{O}}(\underline{\mathrm{r}})=\sum_{\mathrm{j}, \mathrm{k}} \mathrm{c}_{\mathrm{k}, \mathrm{l}}^{\mathrm{O}} \exp \left(\mathrm{i}\left[\mathrm{j} \underline{\mathrm{G}}_{\mathrm{o} 1}{ }^{\prime}+\mathrm{k} \underline{\mathrm{G}}_{\mathrm{o} 2}{ }^{\prime}\right] \mathrm{r}\right), \quad \mathrm{j}, \mathrm{k} \text { integer }
$$

with corresponding reciprocal lattice vectors $\underline{G}_{01}$ ', $\underline{G}_{02}$ ' derived from $\underline{R}_{01}$ ', $\underline{R}_{02}$ '. The lattice vectors $\underline{\mathrm{R}}_{01}, \underline{\mathrm{R}}_{02}$ ' of the overlayer and $\underline{\mathrm{R}}_{\mathrm{o} 1}, \underline{\mathrm{R}}_{\mathrm{o} 2}$ of the substrate are, in general, connected by a linear transformation

$$
\left(\begin{array}{l}
\underline{\mathrm{R}}_{\mathrm{o} 1}{ }^{\prime} \\
\underline{\mathrm{R}}_{\mathrm{o} 2}{ }^{\prime}
\end{array}\right)=\underline{\underline{\mathrm{M}}} \cdot\left(\begin{array}{l}
\underline{\mathrm{R}}_{\mathrm{o} 1} \\
\underline{\mathrm{R}}_{\mathrm{o} 2}
\end{array}\right)
$$

referring to the overlayer periodicity described by matrix $\underline{\underline{M}}$ in $(2 \times 2)$ matrix notation [5]. Then the corresponding reciprocal lattice vectors $\underline{\mathrm{G}}_{01}, \underline{\mathrm{G}}_{02}$, and $\underline{\mathrm{G}}_{\mathrm{o} 1}, \underline{\mathrm{G}}_{02}$ transform according to

$$
\left(\begin{array}{l}
\underline{\mathrm{G}}_{\mathrm{o} 1}{ }^{\prime} \\
\underline{\mathrm{G}}_{\mathrm{o} 2}{ }^{\prime}
\end{array}\right)=\underline{\mathrm{K}} \cdot\left(\begin{array}{l}
\underline{\mathrm{G}}_{\mathrm{o} 1} \\
\underline{\mathrm{G}}_{\mathrm{o} 2}
\end{array}\right)
$$

where the orthogonality relations of real space and reciprocal lattice vectors lead to

$$
\underline{\underline{\mathrm{M}}} \cdot \underline{\underline{\mathrm{K}}}=\underline{\underline{1}} \quad \text { or } \quad \underline{\underline{\mathrm{K}}}=\left(\underline{\underline{\mathrm{M}^{-1}}}\right)^{+}=\left(\underline{\underline{\mathrm{M}^{+}}}\right)^{-1} .
$$

A superposition of the property functions $f_{S}(\underline{r})$ of the substrate and $f_{O}(\underline{r})$ of the overlayer to describe the combined system can always be represented formally by an infinite Fourier series with respect to the substrate lattice $\underline{\mathrm{R}}_{01}, \underline{\mathrm{R}}_{02}$, i.e. by

$$
\mathrm{f}(\underline{\mathrm{r}})=\mathrm{f}_{\mathrm{S}}(\underline{\mathrm{r}})+\mathrm{f}_{\mathrm{O}}(\underline{\mathrm{r}})=\sum_{\mathrm{j}, \mathrm{k}} \mathrm{c}_{\mathrm{j}, \mathrm{k}}^{\mathrm{S}} \exp \left(\mathrm{i}\left[\mathrm{j}_{\mathrm{G} 1}+\mathrm{k} \underline{\mathrm{G}}_{\mathrm{o} 2}\right] \underline{\mathrm{r}}\right) \mathrm{a}_{\mathrm{j}, \mathrm{k}}^{\mathrm{M}}(\underline{\mathrm{r}})
$$

where factors 


$$
\mathrm{a}_{\mathrm{j}, \mathrm{k}}^{\mathrm{M}}(\underline{\mathrm{r}})=1+\frac{\mathrm{c}_{\mathrm{j}, \mathrm{k}}^{\mathrm{O}}}{\mathrm{c}_{\mathrm{j}, \mathrm{k}}^{\mathrm{S}}} \exp \left(\mathrm{i}\left[\mathrm{j}\left(\underline{\mathrm{G}}_{\mathrm{o} 1}{ }^{\prime}-\underline{\mathrm{G}}_{\mathrm{o} 1}\right)+\mathrm{k}\left(\underline{\mathrm{G}}_{\mathrm{o} 2}{ }^{\prime}-\underline{\mathrm{G}}_{\mathrm{o} 2}\right)\right] \underline{\mathrm{r}}\right)
$$

act as modulation functions in the Fourier expansion and are responsible for $\mathrm{f}(\underline{\mathrm{r}})$ not being strictly periodic in the general case. However, these functions $\mathrm{a}_{\mathrm{j}, \mathrm{k}}^{\mathrm{M}}(\underline{\mathrm{r}})$ themselves are periodic and characterized by reciprocal lattice vectors $\underline{\mathrm{G}}_{\mathrm{M} 1}, \underline{\mathrm{G}}_{\mathrm{M} 2}$ where according to (4), (7)

$$
\left(\begin{array}{l}
\underline{\mathrm{G}}_{\mathrm{M} 1} \\
\underline{\mathrm{G}}_{\mathrm{M} 2}
\end{array}\right)=\left(\begin{array}{l}
\underline{\mathrm{G}}_{\mathrm{o} 1}{ }^{\prime}-\underline{\mathrm{G}}_{\mathrm{o} 1} \\
\underline{\mathrm{G}}_{\mathrm{o} 2}{ }^{\prime}-\underline{\mathrm{G}}_{\mathrm{o} 2}
\end{array}\right)=[\underline{\mathrm{K}}-\underline{1}] \cdot\left(\begin{array}{l}
\underline{\mathrm{G}}_{\mathrm{o} 1} \\
\underline{\mathrm{G}}_{\mathrm{o} 2}
\end{array}\right) .
$$

This corresponds to real space periodicity vectors $\underline{\mathrm{R}}_{\mathrm{M} 1}, \underline{\mathrm{R}}_{\mathrm{M} 2}$, called moiré lattice vectors in the following, which result from orthogonality transformations applied to (8) to yield

$$
\left(\begin{array}{l}
\underline{\mathrm{R}}_{\mathrm{M} 1} \\
\underline{\mathrm{R}}_{\mathrm{M} 2}
\end{array}\right)=\underline{\mathrm{P}} \cdot\left(\begin{array}{l}
\underline{\mathrm{R}}_{\mathrm{o} 1} \\
\underline{\mathrm{R}}_{\mathrm{o} 2}
\end{array}\right)
$$

with

$$
\underline{\underline{\mathrm{P}}} \cdot[\underline{\underline{\mathrm{K}}}-\underline{\underline{1}}]^{+}=\underline{\underline{ }}^{1} \quad \text { or } \quad \underline{\underline{\mathrm{P}}}=\left([\underline{\underline{\mathrm{K}}}-\underline{\underline{1}}]^{+}\right)^{-1}=(\underline{\underline{1}}-\underline{\underline{\mathrm{M}}})^{-1} \underline{\underline{\mathrm{M}}} .
$$

If the overlayer lattice vectors $\underline{\mathrm{R}}_{\mathrm{o1}}, \underline{\mathrm{R}}_{\mathrm{o} 2}$ ' are very close to those of the substrate, $\underline{\mathrm{R}}_{\mathrm{o} 1}, \underline{\mathrm{R}}_{\mathrm{o} 2}$, the corresponding reciprocal lattice vectors $\underline{\mathrm{G}}_{01}, \underline{\mathrm{G}}_{\mathrm{o} 2}$ ' and $\underline{\mathrm{G}}_{\mathrm{o} 1}, \underline{\mathrm{G}}_{02}$ are also very similar. Thus, the reciprocal lattice vectors $\underline{G}_{M 1}, \underline{G}_{M 2}$ in (8) become quite small compared with those of the reciprocal overlayer and substrate lattices. As a consequence, the moiré lattice vectors $\underline{R}_{M 1}, \underline{R}_{M 2}$ in real space will, according to (9), (10), be considerably larger than the lattice vectors $\underline{R}_{01}, \underline{R}_{02}$ of the substrate. This introduces long range modulations in the property function $\mathrm{f}(\underline{\mathrm{r}})$ of the combined overlayer / substrate system which are perceived as periodic patterns, described by fictitious lattice vectors $\underline{\mathrm{R}}_{\mathrm{M} 1}, \underline{\mathrm{R}}_{\mathrm{M} 2}$, which reflect the quasi-periodic arrangement of moirons.

\subsection{Moiré lattices of Wood notated overlayers}

The general formalism of obtaining appropriate moiré lattice vectors $\underline{R}_{M 1}, \underline{R}_{M 2}$ can be specified further by using a Wood notation $[4,5]$ to describe the overlayer periodicity. Let us assume the substrate surface to be defined in its periodicity by primitive lattice vectors $\underline{R}_{01}, \underline{R}_{02}$ which may be of different length, given by ratio $\mathrm{q}=\mathrm{R}_{\mathrm{o} 1} / \mathrm{R}_{\mathrm{o} 2}$, and form an angle $\omega$ between them, see Fig. 2 . This co- 
vers all possible 2-dimensional Bravais lattices listed in Table 1. Then an anisotropically scaled and rotated overlayer, in Wood notation denoted as $\left(\mathrm{p}_{1} \mathrm{x}_{\mathrm{p}}\right) \mathrm{R} \alpha$ with the primitive unit cell of the substrate used as reference, will be described by lattice vectors $\underline{\mathrm{R}}_{01}, \underline{\mathrm{R}}_{\mathrm{o} 2}$ ' where simple vector algebra yields, for scaling factors $\mathrm{p}_{1}, \mathrm{p}_{2}$ and rotation angle $\alpha$, a transformation

$$
\begin{gathered}
\left(\begin{array}{l}
\underline{\mathrm{R}}_{\mathrm{o} 1}^{\prime} \\
\underline{\mathrm{R}}_{\mathrm{o} 2}{ }^{\prime}
\end{array}\right)=\frac{1}{\sin (\omega)} \cdot\left(\begin{array}{cc}
\mathrm{p}_{1} \sin (\omega-\alpha) & \mathrm{q} \mathrm{p}_{1} \sin (\alpha) \\
-\frac{1}{\mathrm{q}} \mathrm{p}_{2} \sin (\alpha) & \mathrm{p}_{2} \sin (\omega+\alpha)
\end{array}\right) \cdot\left(\begin{array}{l}
\underline{\mathrm{R}}_{\mathrm{o} 1} \\
\underline{\mathrm{R}}_{\mathrm{o} 2}
\end{array}\right)=\underline{\underline{M}} \cdot\left(\begin{array}{l}
\underline{\mathrm{R}}_{\mathrm{o} 1} \\
\underline{\mathrm{R}}_{\mathrm{o} 2}
\end{array}\right), \\
\mathrm{q}=\frac{\mathrm{R}_{\mathrm{o} 1}}{\mathrm{R}_{\mathrm{o} 2}}
\end{gathered}
$$

connecting the Wood notation with matrix $\underline{\underline{M}}$ of the (2x2) matrix notation used in (3) of Sec. 2.1. Then, depending on the scaling factors $\mathrm{p}_{1}, \mathrm{p}_{2}$ and rotation angle $\alpha$, the superposition of the overlayer and the substrate may give rise to a quasi-periodic moiré pattern described, according to the previous section, by moiré lattice vectors $\underline{R}_{M 1}, \underline{R}_{M 2}$ given by (9), (10) and yielding

$$
\begin{aligned}
\left(\begin{array}{l}
\underline{\mathrm{R}}_{\mathrm{M} 1} \\
\underline{\mathrm{R}}_{\mathrm{M} 2}
\end{array}\right) & =\frac{1}{\Delta \sin (\omega)} \cdot\left(\begin{array}{cc}
\mathrm{p}_{1}\left[\sin (\omega-\alpha)-\mathrm{p}_{2} \sin (\omega)\right] & \mathrm{q} \mathrm{p}_{1} \sin (\alpha) \\
-\frac{1}{\mathrm{q}} \mathrm{p}_{2} \sin (\alpha) & \mathrm{p}_{2}\left[\sin (\omega+\alpha)-\mathrm{p}_{1} \sin (\omega)\right]
\end{array}\right) \cdot\left(\begin{array}{l}
\underline{\mathrm{R}}_{\mathrm{o} 1} \\
\underline{\mathrm{R}}_{\mathrm{o} 2}
\end{array}\right)= \\
& =\underline{\mathrm{P}} \cdot\left(\begin{array}{l}
\underline{\mathrm{R}}_{\mathrm{o} 1} \\
\underline{\mathrm{R}}_{\mathrm{o} 2}
\end{array}\right)
\end{aligned}
$$

with

$$
\Delta=1+\mathrm{p}_{1} \mathrm{p}_{2}-\left(\mathrm{p}_{1}+\mathrm{p}_{2}\right) \cos (\alpha)+\left(\mathrm{p}_{1}-\mathrm{p}_{2}\right) \cot (\omega) \sin (\alpha)
$$

Transformation (12) can be used to define five geometric parameters describing the moiré lattice which can actually be measured. First, the moiré factors $\kappa_{1}, \kappa_{2}$ denote the lengths ratios of the moiré lattice vectors $\underline{\mathrm{R}}_{\mathrm{M} 1}, \underline{\mathrm{R}}_{\mathrm{M} 2}$ and their substrate counterparts $\underline{\mathrm{R}}_{01}, \underline{\mathrm{R}}_{\mathrm{o} 2}$ where according to (12),

$$
\begin{aligned}
& \kappa_{1}=\frac{\mathrm{R}_{\mathrm{M} 1}}{\mathrm{R}_{\mathrm{o} 1}}=\frac{\mathrm{p}_{1}}{\Delta} \sqrt{1+\mathrm{p}_{2}^{2}-2 \mathrm{p}_{2} \cos (\alpha)}, \\
& \kappa_{2}=\frac{\mathrm{R}_{\mathrm{M} 2}}{\mathrm{R}_{\mathrm{o} 2}}=\frac{\mathrm{p}_{2}}{\Delta} \sqrt{1+\mathrm{p}_{1}^{2}-2 \mathrm{p}_{1} \cos (\alpha)} .
\end{aligned}
$$


Thus, moiré factors $\kappa_{1}, \kappa_{2}$ can be understood as lattice constants of the moiré lattice in units of those of the substrate. Second, the moiré angles $\gamma_{1}, \gamma_{2}$ refer to angles spanned by vectors $\underline{R}_{M 1}, \underline{R}_{M 2}$ and their substrate counterparts $\underline{\mathrm{R}}_{01}, \underline{\mathrm{R}}_{02}$, see Fig. 2, and are given by

$$
\begin{aligned}
& \cos \left(\gamma_{1}\right)=\frac{\underline{R}_{\mathrm{M} 1} \underline{R}_{\mathrm{o} 1}}{\mathrm{R}_{\mathrm{M} 1} \mathrm{R}_{\mathrm{o} 1}}=\frac{\cos (\alpha)-\mathrm{p}_{2}}{\sqrt{1+\mathrm{p}_{2}^{2}-2 \mathrm{p}_{2} \cos (\alpha)}}, \tan \left(\gamma_{1}\right)=\frac{\sin (\alpha)}{\left[\cos (\alpha)-\mathrm{p}_{2}\right]}, \\
& \cos \left(\gamma_{2}\right)=\frac{\underline{\mathrm{R}}_{\mathrm{M} 2} \underline{\mathrm{R}}_{\mathrm{o} 2}}{\mathrm{R}_{\mathrm{M} 2} \mathrm{R}_{\mathrm{o} 2}}=\frac{\cos (\alpha)-\mathrm{p}_{1}}{\sqrt{1+\mathrm{p}_{1}^{2}-2 \mathrm{p}_{1} \cos (\alpha)}}, \tan \left(\gamma_{2}\right)=\frac{\sin (\alpha)}{\left[\cos (\alpha)-\mathrm{p}_{1}\right]},
\end{aligned}
$$

Further, the angle $\delta$ spanned by the moiré lattice vectors $\underline{\mathrm{R}}_{\mathrm{M} 1}, \underline{\mathrm{R}}_{\mathrm{M} 2}$ is given by

$$
\begin{aligned}
& \cos (\delta)=\frac{\underline{R}_{M 1} \underline{R}_{M 2}}{R_{M 1} R_{M}}=\frac{\left\lfloor 1+p_{1} p_{2}-\left(p_{1}+p_{2}\right) \cos (\alpha)\right\rfloor \cos (\omega)+\left(p_{2}-p_{1}\right) \sin (\alpha) \sin (\omega)}{\sqrt{\left(1+p_{1}^{2}-2 p_{1} \cos (\alpha)\right)\left(1+p_{2}^{2}-2 p_{2} \cos (\alpha)\right)}} \\
& \tan (\delta)=\frac{\Delta \cdot \tan (\omega)}{1+p_{1} p_{2}-\left(p_{1}+p_{2}\right) \cos (\alpha)+\left(p_{2}-p_{1}\right) \tan (\omega) \sin (\alpha)}
\end{aligned}
$$

It must be emphasized that the present theoretical treatment of quasi-periodic interference patterns at surfaces described by moiré lattices is based exclusively on the interplay of overlayer and substate periodicities and ignores exact geometric details of the local atom arrangement. In particular, the general results are independent of the actual atom basis of the overlayer. As such, the formalism can be applied to overlayers with both monoatomic and polyatomic atom bases where, so far, experimental evidence has focused on polyatomic systems represented by graphene or h-BN overlayers.

\subsection{Moirons at overlayers forming coincidence lattices}

The moiron arrangement at general $\left(\mathrm{p}_{1} \mathrm{x}_{2}\right) \mathrm{R} \alpha$ overlayers will not be strictly periodic, with moirons varying slightly in shape at different sites, and the description of their positions by moré lattices (12) can only be approximate. However, the combined overlayer / substrate surface may form an altogether commensurate phase with a coincidence lattice described by lattice vectors $\underline{R}_{1}$, $\underline{\mathrm{R}}_{2}$ resulting in a (possibly large) supercell which is shared by the overlayer and the substrate. Thus, 
vectors $\underline{R}_{1}, \underline{R}_{2}$ are integer valued linear combinations of the lattice vectors of both the substrate and the overlayer, i.e.

$$
\left(\begin{array}{l}
\underline{\mathrm{R}}_{1} \\
\underline{\mathrm{R}}_{2}
\end{array}\right)=\underline{\underline{\mathrm{m}}} \cdot\left(\begin{array}{l}
\underline{\mathrm{R}}_{\mathrm{o} 1}^{\prime} \\
\underline{\mathrm{R}}_{\mathrm{o} 2}^{\prime}
\end{array}\right)=\underline{\mathrm{m}}^{\prime} \cdot\left(\begin{array}{l}
\underline{\mathrm{R}}_{\mathrm{o} 1} \\
\underline{\mathrm{R}}_{\mathrm{o} 2}
\end{array}\right)
$$

with $\underline{\underline{m}}$ and $\underline{\underline{m}}^{\prime}$ denoting integer valued (2x2) matrices defining the coincidence lattice vectors $\underline{\mathrm{R}}_{1}$, $\underline{\mathrm{R}}_{2}$ by those of the overlayer and substrate, respectively. This leads to a transformation between the lattice vectors of the overlayer and substrate given by

$$
\left(\begin{array}{l}
\underline{\mathrm{R}}_{\mathrm{o} 1}^{\prime} \\
\underline{\mathrm{R}}_{\mathrm{o} 2}^{\prime}
\end{array}\right)=\left(\underline{\underline{\mathrm{m}}}^{-1} \cdot \underline{\mathrm{m}}^{\prime}\right) \cdot\left(\begin{array}{l}
\underline{\mathrm{R}}_{\mathrm{o} 1} \\
\underline{\mathrm{R}}_{\mathrm{o} 2}
\end{array}\right)=\underline{\underline{\mathrm{M}}} \cdot\left(\begin{array}{l}
\underline{\mathrm{R}}_{\mathrm{o} 1} \\
\underline{\mathrm{R}}_{\mathrm{o} 2}
\end{array}\right)
$$

where the (2x2) matrix $\underline{\underline{M}}$, appearing also in (3), (11), contains only integer or rational elements $\mathrm{M}_{\mathrm{ij}}$ as expected for coincidence lattices [5].

Obviously, moiron arrangements at surfaces with coincidence lattice geometry will be strictly periodic with periodicity vectors given by $\underline{\mathrm{R}}_{1}, \underline{\mathrm{R}}_{2}$. Corresponding moiré lattice vectors $\underline{\mathrm{R}}_{M 1}, \underline{\mathrm{R}}_{\mathrm{M} 2}$ are then defined according to (9), (10), (19), (20) by

$$
\left(\begin{array}{l}
\underline{\mathrm{R}}_{\mathrm{M} 1} \\
\underline{\mathrm{R}}_{\mathrm{M} 2}
\end{array}\right)=(\underline{\underline{1}}-\underline{\underline{\mathrm{M}}})^{-1} \underline{\underline{\mathrm{M}}} \cdot\left(\begin{array}{l}
\underline{\mathrm{R}}_{\mathrm{o} 1} \\
\underline{\mathrm{R}}_{\mathrm{o} 2}
\end{array}\right)=\left(\underline{\underline{\mathrm{m}}}-\underline{\underline{\mathrm{m}}}^{\prime}\right)^{-1} \underline{\mathrm{m}}^{\prime} \cdot\left(\begin{array}{l}
\underline{\mathrm{R}}_{\mathrm{o} 1} \\
\underline{\mathrm{R}}_{\mathrm{o} 2}
\end{array}\right) \text {. }
$$

Applying relation (19) this leads to

$$
\left(\begin{array}{l}
\underline{\mathrm{R}}_{1} \\
\underline{\mathrm{R}}_{2}
\end{array}\right)=\underline{\mathrm{m}}^{\prime} \cdot\left(\begin{array}{l}
\underline{\mathrm{R}}_{\mathrm{o} 1} \\
\underline{\mathrm{R}}_{\mathrm{o} 2}
\end{array}\right)=\left(\underline{\underline{\mathrm{m}}}-\underline{\underline{\mathrm{m}}}^{\prime}\right) \cdot\left(\begin{array}{l}
\underline{\mathrm{R}}_{\mathrm{M} 1} \\
\underline{\mathrm{R}}_{\mathrm{M} 2}
\end{array}\right) .
$$

where ( $\left.\underline{\underline{\mathrm{m}}}-\underline{\underline{\mathrm{m}}}^{\prime}\right)$ is an integer valued ( $\left.2 \times 2\right)$ matrix. Thus, the coincidence lattice vectors $\underline{\mathrm{R}}_{1}, \underline{\mathrm{R}}_{2}$ are given by integer valued linear combinations of the moiré lattice vectors $\underline{R}_{M 1}, \underline{R}_{M 2}$ and the moiré lattice vectors, while not describing a strict periodicity of the surface, can serve as a basis to define the coincidence lattice. Further, relation (22) can be used to show that the unit cell area A of the coincidence lattice is an integer multiple of the unit cell area $\mathrm{A}_{\text {moiré }}$ of the moiré lattice where

$$
A=\operatorname{det}\left(\underline{\underline{m}}-\underline{\underline{m^{\prime}}}\right) \cdot A_{\text {moiré }}
$$

Since each moiré unit cell contains exactly one moiron the unit cell of the coincidence lattice will always include $\mathrm{N}$ moirons where according to (23) 


$$
\mathrm{N}=\frac{\mathrm{A}}{\mathrm{A}_{\text {moiré }}}=\operatorname{det}\left(\underline{\underline{\mathrm{m}}}-\underline{\underline{\mathrm{m}^{\prime}}}\right)
$$

A comparison of transformation (20) with (11) shows immediately that for general scaled and rotated $\left(\mathrm{p}_{1} \times \mathrm{p}_{2}\right) \mathrm{R} \alpha$ overlayers scaling factors $\mathrm{p}_{1}, \mathrm{p}_{2}$ and rotation angles $\alpha$ are restricted to specific discrete values to yield exact coincidence lattices since the matrix elements $M_{i j}$ in (11) will in general be real rather than rational valued. However, real valued elements $\mathrm{M}_{\mathrm{ij}}$ can be approximated to any degree of accuracy by rational numbers. Therefore, it is always possible to determine approximate coincidence lattices for a given accuracy if their lattice vectors are chosen large enough.

As an illustration, we mention ( $\mathrm{p}$ x p) R $\alpha$ coincidence lattices appearing for hexagonal overlayers on hexagonal substrate. Assuming an acute representation of hexagonal lattice vectors $\underline{\mathrm{R}}_{\mathrm{o} 1}, \underline{\mathrm{R}}_{\mathrm{o} 2}$, i. e. $\omega=\angle\left(\underline{\mathrm{R}}_{\mathrm{o} 1}, \underline{\mathrm{R}}_{\mathrm{o} 2}\right)=60^{\circ}$, transformation matrices $\underline{\underline{\mathrm{m}}}, \underline{\underline{\mathrm{m}}}$ ' are found to be in general determined by four integer numbers $a, b, a^{\prime}, b^{\prime}$ (without proof) where

$$
\underline{\underline{m}}=\left(\begin{array}{cc}
a & b \\
-b & a+b
\end{array}\right), \quad \underline{\underline{m^{\prime}}}=\left(\begin{array}{cc}
a^{\prime} & b^{\prime} \\
-b^{\prime} & a^{\prime}+b^{\prime}
\end{array}\right)
$$

and thus transformation matrix $\underline{\underline{M}}$ in (20) is given by

$$
\underline{\underline{M}}=\left(\underline{\underline{m}}^{-1} \cdot \underline{\underline{m}}^{\prime}\right)=\frac{1}{\Gamma} \cdot\left(\begin{array}{cc}
a a^{\prime}+b b^{\prime}+b a^{\prime} & a b^{\prime}-b a^{\prime} \\
b a^{\prime}-a b^{\prime} & a a^{\prime}+b b^{\prime}+a b^{\prime}
\end{array}\right)
$$

with

$$
\Gamma=a^{2}+b^{2}+a b
$$

Therefore, possible scaling factors $\mathrm{p}$ are given by

$$
p=\frac{R_{o 1}^{\prime}}{R_{o 1}}=\sqrt{\frac{\left(a^{\prime 2}+b^{\prime 2}+a^{\prime} b^{\prime}\right)}{\left(a^{2}+b^{2}+a b\right)}}
$$

and rotation angles $\alpha$ by

$$
\cos (\alpha)=\frac{\underline{\mathrm{R}}_{\mathrm{o} 1} \underline{\mathrm{R}}_{\mathrm{o} 1}}{\mathrm{R}_{\mathrm{o} 1}{ }^{\prime} \mathrm{R}_{\mathrm{o} 1}}=\frac{1}{2 \mathrm{p} \Gamma}\left[2 \mathrm{a}^{\prime} \mathrm{a}+2 \mathrm{~b}^{\prime} \mathrm{b}+\mathrm{a}^{\prime} \mathrm{b}+\mathrm{b}^{\prime} \mathrm{a}\right] .
$$

This shows, in particular, the discreteness of possible rotation angles $\alpha$. As a result, possible moiré lattice vectors of ( $\mathrm{p} \times \mathrm{p}$ ) R $\alpha$ overlayers can be determined according to (9), (10) to yield 


$$
\left(\begin{array}{l}
\underline{\mathrm{R}}_{\mathrm{M} 1} \\
\underline{\mathrm{R}}_{\mathrm{M} 2}
\end{array}\right)=\frac{1}{\Lambda} \cdot\left(\begin{array}{cc}
\left(\mathrm{a}^{\prime}+\mathrm{b}^{\prime}\right)\left(\mathrm{b}-\mathrm{b}^{\prime}\right)+\mathrm{a}^{\prime}\left(\mathrm{a}-\mathrm{a}^{\prime}\right) & \mathrm{a} \mathrm{b}^{\prime}-\mathrm{b} \mathrm{a}^{\prime} \\
\mathrm{b} \mathrm{a}^{\prime}-\mathrm{ab}^{\prime} & \left(\mathrm{a}^{\prime}+\mathrm{b}^{\prime}\right)\left(\mathrm{a}-\mathrm{a}^{\prime}\right)+\mathrm{b}^{\prime}\left(\mathrm{b}-\mathrm{b}^{\prime}\right)
\end{array}\right) \cdot\left(\begin{array}{l}
\underline{\mathrm{R}}_{\mathrm{o} 1} \\
\underline{\mathrm{R}}_{\mathrm{o} 2}
\end{array}\right)
$$

with

$$
\Lambda=\left(a^{\prime}-a\right)^{2}+\left(b^{\prime}-b\right)^{2}+\left(a^{\prime}-a\right)\left(b^{\prime}-b\right)
$$

Relation (29) shows also that, while rotation angles $\alpha$ for exact coincidence lattices are discrete, their distribution is quasi-continuous if large enough integers a, b, a', b', reflecting possibly very large coincidence supercells, are considered.

\section{RESULTS AND DISCUSSION}

In this section we discuss results from the general formalism detailed in Section 2 with emphasis on surface systems where moiré patterns have been observed in experiment. These include, with increasing complexity, isotropically scaled (p x p), Sec. 3.1, rotated (1x1) R $\alpha$, Sec. 3.2, rotated and isotropically scaled (p x p) $\mathrm{R} \alpha$, Sec. 3.3, and general ( $\left.\mathrm{p}_{1} \times \mathrm{p}_{2}\right) \mathrm{R} \alpha$ overlayers, Sec. 3.4.

\subsection{Isotropically scaled ( $\mathbf{p} \times \mathbf{p})$ overlayers}

Moiré patterns have been observed for graphene overlayers on a number of hexagonal substrate surfaces where an isotropically scaled ( $\mathrm{p}$ x p) structure without rotation has been discussed. Table 2 lists corresponding scaling factors $\mathrm{p}$ determined from ratios of the graphene lattice constant and those of the metal surfaces. As an example, Fig. 3 shows a simulation of a graphene overlayer at the $\operatorname{Pt}(111)$ surface where $\mathrm{p}=0.89$. Here a hexagonal moiré lattice corresponding to a lattice constant $\mathrm{a}_{\text {moiré }}=8 \mathrm{aPt}_{\mathrm{Pt}}$ is observed [20].

The theoretical treatment of moiré interference of isotropically scaled ( $\mathrm{p}$ x p) overlayers can be obtained from the general formalism of Sec. 2 by setting $p_{1}=p_{2}=p$ and $\alpha=0^{\circ}$. This yields for the transformation between overlayer and substrate lattice vectors of general Bravais lattices according to $(11)$

$$
\left(\begin{array}{l}
\underline{\mathrm{R}}_{\mathrm{o} 1}^{\prime} \\
\underline{\mathrm{R}}_{\mathrm{o} 2}{ }^{\prime}
\end{array}\right)=\left(\begin{array}{ll}
\mathrm{p} & 0 \\
0 & \mathrm{p}
\end{array}\right) \cdot\left(\begin{array}{l}
\underline{\mathrm{R}}_{\mathrm{o} 1} \\
\underline{\mathrm{R}}_{\mathrm{o} 2}
\end{array}\right)=\mathrm{p} \cdot\left(\begin{array}{l}
\underline{\mathrm{R}}_{\mathrm{o} 1} \\
\underline{\mathrm{R}}_{\mathrm{o} 2}
\end{array}\right),
$$


and hence for the moiré lattice vectors $\underline{R}_{M 1}, \underline{R}_{M 2}$ according to (12), (13)

$$
\left(\begin{array}{l}
\underline{\mathrm{R}}_{\mathrm{M} 1} \\
\underline{\mathrm{R}}_{\mathrm{M} 2}
\end{array}\right)=\left(\begin{array}{cc}
\frac{\mathrm{p}}{1-\mathrm{p}} & 0 \\
0 & \frac{\mathrm{p}}{1-\mathrm{p}}
\end{array}\right) \cdot\left(\begin{array}{l}
\underline{\mathrm{R}}_{\mathrm{o} 1} \\
\underline{\mathrm{R}}_{\mathrm{o} 2}
\end{array}\right)=\frac{\mathrm{p}}{1-\mathrm{p}} \cdot\left(\underline{\mathrm{R}}_{\mathrm{o} 1}\right) \text {. }
$$

Further, the moiré parameters given by (14), (15), (16), (17) simplify to yield

$$
\begin{aligned}
& \kappa_{i}=\frac{R_{M i}}{R_{o i}}=\kappa=\frac{p}{|1-p|} \quad \\
& \gamma_{i}=\gamma=\left\{\begin{array}{ll}
0^{\circ} & \text { for } p<1 \\
180^{\circ} & \text { for } p>1
\end{array} \quad \mathrm{i}=1,2 .\right.
\end{aligned}
$$

This shows that isotropic overlayer scaling without rotation always results in moiré lattice vectors $\underline{\mathrm{R}}_{\mathrm{M} 1}, \underline{\mathrm{R}}_{\mathrm{M} 2}$ that point along the directions of the initial surface basis vectors $\underline{\mathrm{R}}_{\mathrm{o}}, \underline{\mathrm{R}}_{\mathrm{02}}$. Thus, the moiré lattice is of the same Bravais lattice type as the substrate. In the case of the experimentally studied hexagonal surfaces this confirms the observed hexagonal moiré patterns. Further, relations (33), (34) show that moiré lattice vectors diverge in their lengths when $p$ approaches $p=1$ which corresponds to lattice constants of the overlayer and substrate lattices that are very close. In this limit the moiron arrangement will become very open with extremely large moirons that may not be observable. This is consistent with experimental findings for graphene overlayers on $\mathrm{Ni}(111)$ substrate [29] where the surface lattice constants differ by less that $1 \%$.

Obviously, moiré lattice vectors may be determined mathematically for any scaling factor $\mathrm{p}>0$ using transformations (33). However, moiré patterns will be clearly detectable only for $\kappa$ values above a minimum size $\kappa_{\mathrm{o}}$ when moirons are large enough to show as separated entities. According to (34), this sets a range of scaling factors $\mathrm{p}$ resulting in visible moiré patterns where after some algebra

$$
\frac{\kappa_{\mathrm{o}}}{\kappa_{\mathrm{o}}+1} \leq \mathrm{p} \leq \frac{\kappa_{\mathrm{o}}}{\kappa_{\mathrm{o}}-1}
$$

Here values $\kappa_{\mathrm{o}}$ close to 5 are reasonable from experience such that scaling factors between 0.8 and 1.2 qualify for clearly visible moiré patterns. 
As discussed in the introduction, graphene on $\mathrm{Ru}(0001)$ substrate forms a coincidence lattice with a supercell of $(25 \times 25)$ carbon honeycombs on a $(23 \times 23)$ supercell of the substrate [6] which leads to a ( $\mathrm{p} \mathrm{x} \mathrm{p)} \mathrm{structure} \mathrm{with} \mathrm{p}=23 / 25=0.92$. According to the coincidence lattice treatment in Sec. 2.3, cp. (19), this is described by superlattice matrices

$$
\underline{\underline{\mathrm{m}}}=\left(\begin{array}{cc}
25 & 0 \\
0 & 25
\end{array}\right), \quad \underline{\underline{m}}^{\prime}=\left(\begin{array}{cc}
23 & 0 \\
0 & 23
\end{array}\right)
$$

and, thus, with (24) the $(23 \times 23)$ supercell of the substrate contains four translationally nonequivalent moirons. On the other hand, graphene on $\operatorname{Pt}(111)$ substrate forms a coincidence lattice with a supercell of $(9 \times 9)$ carbon honeycombs on a $(8 \times 8)$ supercell of the substrate [20] which leads to $\mathrm{p}=8 / 9=0.89$ reflecting superlattice matrices

$$
\underline{\underline{\mathrm{m}}}=\left(\begin{array}{ll}
9 & 0 \\
0 & 9
\end{array}\right), \quad \underline{\underline{\mathrm{m}^{\prime}}}=\left(\begin{array}{ll}
8 & 0 \\
0 & 8
\end{array}\right)
$$

and, as a result, the supercell of the substrate contains exactly one moiron. Thus, all moirons of the moiré lattice are identical and the coincidence lattice agrees with the moiré lattice.

\subsection{Rotated ( 1 x 1$)$ Ra overlayers}

Moiré patterns have been found in experiments for graphene overlayers on graphene where a rotated ( $1 \times 1) \mathrm{R} \alpha$ structure without scaling (but possibly combined with lateral translation) has been discussed [13-16]. As an illustration, Fig. 4 shows a simulation of a (hexagonal) graphene overlayer on graphene substrate for a rotation angle $\alpha=3.5^{\circ}$ claimed by experiment [16]. This is found to exhibit a hexagonal pattern with a moiré lattice corresponding to a lattice constant $\mathrm{a}_{\text {moiré }}=$ 16.4 agraphene as indicated in Fig. 4 by the moiré lattice vectors $\underline{R}_{M 1}, \underline{R}_{M 2}$.

The theoretical treatment of moiré interference of rotated ( $1 \times 1) \mathrm{R} \alpha$ overlayers is obtained from the general formalism of Sec. 2 by setting $\mathrm{p}_{1}=\mathrm{p}_{2}=1$. This yields for the transformation between overlayer and substrate lattice vectors of general Bravais lattices according to (11)

$$
\left(\begin{array}{l}
\underline{\mathrm{R}}_{\mathrm{o} 1}{ }^{\prime} \\
\underline{\mathrm{R}}_{\mathrm{o} 2}{ }^{\prime}
\end{array}\right)=\frac{1}{\sin (\omega)} \cdot\left(\begin{array}{cc}
\sin (\omega-\alpha) & \mathrm{q} \sin (\alpha) \\
-\frac{1}{\mathrm{q}} \sin (\alpha) & \sin (\omega+\alpha)
\end{array}\right) \cdot\left(\begin{array}{l}
\underline{\mathrm{R}}_{\mathrm{o} 1} \\
\underline{\mathrm{R}}_{\mathrm{o} 2}
\end{array}\right), \quad \mathrm{q}=\frac{\mathrm{R}_{\mathrm{o} 1}}{\mathrm{R}_{\mathrm{o} 2}}
$$


and hence for the moiré lattice vectors $\underline{\mathrm{R}}_{\mathrm{M} 1}, \underline{\mathrm{R}}_{\mathrm{M} 2}$ according to (12), (13)

$$
\left(\begin{array}{l}
\underline{\mathrm{R}}_{\mathrm{M} 1} \\
\underline{\mathrm{R}}_{\mathrm{M} 2}
\end{array}\right)=\chi \cdot\left(\begin{array}{cc}
{[\sin (\omega-\alpha)-\sin (\omega)]} & \mathrm{q} \sin (\alpha) \\
-\frac{1}{\mathrm{q}} \sin (\alpha) & {[\sin (\omega+\alpha)-\sin (\omega)]}
\end{array}\right) \cdot\left(\begin{array}{l}
\underline{\mathrm{R}}_{\mathrm{o} 1} \\
\underline{\mathrm{R}}_{\mathrm{o} 2}
\end{array}\right)
$$

with

$$
\chi=\frac{1}{2 \sin (\omega)[1-\cos (\alpha)]}
$$

Further, the moiré parameters given by (14), (15), (16), (17) simplify to yield

$$
\begin{aligned}
& \kappa_{\mathrm{i}}=\frac{\mathrm{R}_{\mathrm{Mi}}}{\mathrm{R}_{\mathrm{oi}}}=\kappa=\left(2\left|\sin \frac{\alpha}{2}\right|\right)^{-1}, \\
& \gamma_{\mathrm{i}}=\gamma=90^{\circ}+\frac{\alpha}{2} \quad \mathrm{i}=1,2 .
\end{aligned}
$$

Relation (43) shows that moiré lattice vectors $\underline{\mathrm{R}}_{\mathrm{M} 1}, \underline{\mathrm{R}}_{\mathrm{M} 2}$ will in general not point along directions of the initial surface basis vectors $\underline{\mathrm{R}}_{01}, \underline{\mathrm{R}}_{02}$. However, both vectors $\underline{\mathrm{R}}_{\mathrm{M} 1}, \underline{\mathrm{R}}_{\mathrm{M} 2}$ are stretched and rotated equally compared with $\underline{\mathrm{R}}_{01}, \underline{\mathrm{R}}_{02}$ such that the moiré lattice is of the same Bravais lattice type as the substrate. This leads for graphene overlayers on graphene substrate always to hexagonal moiré patterns as observed in the experiment. Relation (42) evidences that moiré lattice vectors diverge in their lengths with rotation angle $\alpha$ approaching zero where for very small $\alpha$ values (42) can be approximated by

$$
\kappa \approx \frac{1}{|\alpha|}
$$

Moiré patterns resulting from rotated (1x1) R $\alpha$ overlayers will be clearly detectable only for $\kappa$ values above a minimum size $\kappa_{\mathrm{o}}$ when moirons are large enough to be discriminated visually. According to (42), this limits rotation angles $\alpha$ to values

$$
\left|\sin \frac{\alpha}{2}\right| \leq \frac{1}{2 \kappa_{\mathrm{o}}}
$$

where reasonable values $\kappa_{\mathrm{o}}$ near 5 yield angles $\alpha$ between $0^{\circ}$ and $10^{\circ}$ for clearly visible moiré patterns. 
Moiré patterns are also affected by lateral shifts of the overlayer. A global shift vector $\underline{\mathrm{v}}$ translating the otherwise rigid overlayer can always be represented by primitive lattice vectors of the substrate as

$$
\underline{\mathrm{V}}=\mathrm{x}_{1} \underline{\mathrm{R}}_{\mathrm{o} 1}+\mathrm{x}_{2} \underline{\mathrm{R}}_{\mathrm{o} 2}, \quad 0 \leq \mathrm{x}_{\mathrm{i}}<1
$$

This leads to a shift of all moirons by a global vector $\underline{\mathrm{V}}$ which according to Fourier theory discussed in Sec. 2 can be shown to be given by

$$
\underline{\mathrm{V}}=\mathrm{x}_{1} \underline{\mathrm{R}}_{\mathrm{M} 1}+\mathrm{x}_{2} \underline{\mathrm{R}}_{\mathrm{M} 2}
$$

Since moiré lattice vectors $\underline{\mathrm{R}}_{\mathrm{M} 1}, \underline{\mathrm{R}}_{\mathrm{M} 2}$ will in general not point along directions of the initial surface basis vectors $\underline{\mathrm{R}}_{01}, \underline{\mathrm{R}}_{02}$ vectors $\underline{\mathrm{V}}$ and $\underline{\mathrm{V}}$ will also point in different directions. Thus, global shifts applied to an overlayer will in general shift the corresponding moiron array along a different direction. The angle $\beta$ between the shift vectors is determined using (39), (40) to yield

$$
\cos (\beta)=\frac{\underline{\mathrm{V}} \underline{\mathrm{v}}}{\overline{\mathrm{V} \mathrm{v}}}=\cos (\gamma)
$$

Thus, angle $\beta$ agrees with the moiré angle $\gamma$ given in (43). Further, the length of vector $\underline{\mathrm{V}}$ amounts, according to (40), (42), to

$$
V=|\underline{V}|=\kappa \sqrt{x_{1}^{2}+\frac{1}{q^{2}} x_{2}^{2}+\frac{2}{q} x_{1} x_{2} \cos (\omega)} \quad R_{o 1}=\kappa v
$$

Therefore, the initial shift vector $\underline{\mathrm{v}}$ applied to the overlayer moves the moiré pattern by a vector $\underline{\mathrm{V}}$ which, according to (49), is larger in length by factor $\kappa$ compared with the initial shift vector $\underline{v}$ and the two vectors are not parallel to each other for $\alpha \neq 0$, see (48).

As an illustration, Fig. 5 shows moiré patterns of the graphene overlayer on graphene substrate for a rotation angle $\alpha=3.5^{\circ}$ discussed above, however, with additional overlayer shifts of $\underline{v}=-1 / 3$ $\underline{\mathrm{R}}_{02}$ (Fig. 5a) and $\underline{\mathrm{v}}=-2 / 3 \underline{\mathrm{R}}_{\mathrm{o} 2}$ (Fig. 5b) applied. According to (43), (48) the angle $\beta$ between vectors $\underline{\mathrm{V}}$ and $\underline{\mathrm{v}}$ amounts to $91.75^{\circ}$ such that the shift of the moirons occurs almost perpendicular to that of the overlayer which is obvious in Fig. 5. Further, the rather small overlayer shifts $\underline{v}$ shown in Fig. 5 lead to quite large moiron shifts $\underline{\mathrm{V}}$ due to the amplification by factor $\kappa=16.4$, see (49).

It should be noted that the moiron shift behaviour is completely analogous for isotropically scaled overlayers with the relations (49), (48) being identical. As an example, scaled (p x p) 
overlayers without rotation yield for the moiré angle $\gamma$ values of $0^{\circ}(\mathrm{p}<1)$ or $180^{\circ}(\mathrm{p}>1)$, see (35). Thus, for these overlayers moirons shifts occur always parallel or anti-parallel to those of the overlayer but will be enhanced by a factor $\kappa$. This result is relevant for an understanding of moiré patterns of graphene near monoatomic steps at metal surfaces. Scanning tunneling microscopy experiments for graphen on $\operatorname{Ir}(111)$ substrate have shown [19] that carbon overlayers can spread over monoatomic steps of the substrate with their basic honeycomb structure remaining intact. Here adjacent terraces offer identical periodicity and substrate lattice vectors while the origins of their lateral atom arrangements differ. This leads to a global lateral shift in the atom arrangement between the adjacent terraces which affects their interference with the overlayer and explains the observed moiron shifts [19]. As an illustration, Fig. 6 shows the simulation of a moiré pattern for graphene spreading over a monoatomic step at the $\operatorname{Ir}(111)$ surface. Here the Ir atoms at adjacent terraces experience a lateral shift of $-\underline{\mathrm{v}}=\left(\underline{\mathrm{R}}_{01}-2 \underline{\mathrm{R}}_{02}\right) / 3$ (corresponding to a relative shift $\underline{\mathrm{v}}$ of the graphene layer) which according to (35), (47) results in a parallel shift $\underline{\mathrm{V}}=\left(\underline{\mathrm{R}}_{\mathrm{M} 1}-2 \underline{\mathrm{R}}_{\mathrm{M} 2}\right) / 3$ of the moiron arrangement between the terraces which is obvious from Fig. 6.

\subsection{Rotated and isotropically scaled ( $\mathbf{p} \times \mathbf{p}) \mathrm{R} \alpha$ overlayers}

The theoretical treatment of ( $\mathrm{p} \times \mathrm{p}$ ) $\mathrm{R} \alpha$ overlayers combines isotropical scaling with rotation and has been considered in experiments for graphene overlayers on hexagonal boron nitride (h-BN) layers. As an example, Fig. 7 shows a simulation of graphene on h-BN where a difference in the lattice constants of $2 \%$, resulting in $p=0.98$, and a rotation of $\alpha=5.4^{\circ}$ is assumed from experiment [30]. Here the hexagonal moiré lattice corresponding to a lattice constant $\mathrm{a}_{\text {moiré }}=10.3 \mathrm{a}_{\mathrm{BN}}$ is evident.

Moiré interference of rotated and isotropically scaled ( $\mathrm{p} \times \mathrm{p}$ ) $\mathrm{R} \alpha$ overlayers are decribed by the general formalism of Sec. 2 by setting $\mathrm{p}_{1}=\mathrm{p}_{2}=\mathrm{p}$. This yields for the transformation between overlayer and substrate lattice vectors of general Bravais lattices according to (11)

$$
\left(\begin{array}{l}
\underline{\mathrm{R}}_{\mathrm{o} 1}^{\prime} \\
\underline{\mathrm{R}}_{\mathrm{o} 2}{ }^{\prime}
\end{array}\right)=\frac{\mathrm{p}}{\sin (\omega)} \cdot\left(\begin{array}{cc}
\sin (\omega-\alpha) & \mathrm{q} \sin (\alpha) \\
-\frac{1}{\mathrm{q}} \sin (\alpha) & \sin (\omega+\alpha)
\end{array}\right) \cdot\left(\begin{array}{l}
\underline{\mathrm{R}}_{\mathrm{o} 1} \\
\underline{\mathrm{R}}_{\mathrm{o} 2}
\end{array}\right), \quad \mathrm{q}=\frac{\mathrm{R}_{\mathrm{o} 1}}{\mathrm{R}_{\mathrm{o} 2}}
$$

and hence for the moiré lattice vectors $\underline{R}_{M 1}, \underline{R}_{M 2}$ according to (12), (13) 


$$
\left(\begin{array}{l}
\underline{\mathrm{R}}_{\mathrm{M} 1} \\
\underline{\mathrm{R}}_{\mathrm{M} 2}
\end{array}\right)=\frac{\mathrm{p}}{\Delta \sin (\omega)} \cdot\left(\begin{array}{cc}
{[\sin (\omega-\alpha)-\mathrm{p} \sin (\omega)]} & \mathrm{q} \sin (\alpha) \\
-\frac{1}{\mathrm{q}} \sin (\alpha) & {[\sin (\omega+\alpha)-\mathrm{p} \sin (\omega)]}
\end{array}\right) \cdot\left(\begin{array}{l}
\underline{\mathrm{R}}_{\mathrm{o} 1} \\
\underline{\mathrm{R}}_{\mathrm{o} 2}
\end{array}\right)
$$

with

$$
\Delta=1+\mathrm{p}^{2}-2 \mathrm{p} \cos (\alpha)
$$

Further, the moiré parameters given by (14), (15), (16), (17) simplify to yield

$$
\begin{aligned}
& \kappa_{i}=\frac{R_{M i}}{R_{o i}}=\kappa=\frac{p}{\sqrt{1+p^{2}-2 p \cos (\alpha)}}, \\
& \cos \left(\gamma_{i}\right)=\frac{R_{M i} \underline{R}_{o 1}}{R_{M i} R_{o 1}}=\cos (\gamma)=\frac{\cos (\alpha)-p}{\sqrt{1+p^{2}-2 p \cos (\alpha)}} \quad i=1,2, \\
& \tan (\gamma)=\frac{\sin (\alpha)}{[\cos (\alpha)-p]} .
\end{aligned}
$$

Fig. 8 gives a perspective view of the moiré angle $\gamma$ in its dependence on the rotation angle $\alpha$ and scaling factor $p$ defined by (54), (55). As discussed earlier, rotation angle $\alpha=0^{\circ}$ and scaling factors $\mathrm{p} \neq 1$, which correspond to scaled ( $\mathrm{p} \times \mathrm{p}$ ) overlayers, lead to a discontinuous behavior with $\gamma$ $=0^{\circ}$ for $\mathrm{p}<1$ and $\gamma=180^{\circ}$ for $\mathrm{p}>1$, see (35) of Sec. 3.1, where the moiré lattice vectors $\underline{\mathrm{R}}_{\mathrm{M} 1}, \underline{\mathrm{R}}_{\mathrm{M} 2}$ always point along the directions of the initial surface basis vectors $\underline{R}_{01}, \underline{R}_{02}$. For very small rotation angles $\alpha$, where $\sin (\alpha)$ and $\cos (\alpha)$ in (55) can be approximated by $\alpha$ and $1-\alpha^{2} / 2$ (in radian units), respectively, we obtain with the small angle expansion for $\sin (\gamma)$

$$
\gamma \approx \begin{cases}\frac{\alpha}{1-p} & \text { for } p<1 \\ 180^{\circ}-\frac{\alpha}{p-1} & \text { for } p>1\end{cases}
$$

This shows that for scaling factors $\mathrm{p}$ approaching $\mathrm{p}=1$ from below (i. e. for overlayer lattice constants below the substrate value but differing by only little from it) the moiré angle $\gamma$ can become much larger than the initial inter-layer rotation angle $\alpha$. Analogous, for $\mathrm{p}$ approaching $\mathrm{p}=1$ from above angles $\gamma$ vary also quite strongly for small rotations $\alpha$. This is obvious from Fig. 8 and illustrated for graphene on h-BN where the lattice constants differ by only $2 \%$ corresponding to $\mathrm{p}=$ 
0.98. For a rotation of only $\alpha=5.4^{\circ}$ [30] we obtain for the moiré angle according to (55) a value $\gamma=$ $80.6^{\circ}$.

Fig. 9 shows the moiré factor $\kappa$ as a function of $\alpha$ and $p$ as defined by (53). Obviously, factor $\kappa$, which characterizes the lattice constant of the moiré pattern, can become rather large for small rotation angles $\alpha$ and scaling factors $\mathrm{p}$ approaching $\mathrm{p}=1$ which is mathematically evident from relation (53) and confirmed by Fig. 9. For small values $\alpha$ and scaling factors $p$ approaching $p=1$ we obtain from (53) approximately

$$
\kappa \approx \frac{p}{\sqrt{(1-p)^{2}+p \alpha^{2}}}
$$

which, setting $\alpha=0$, yields relation (34) while for $p=1$ the approximate relation (44) is obtained.

As another important result we note that relations (53), (54), (55) determining the moiré parameters $\kappa$ and $\gamma$ do not require values for $\mathrm{q}, \omega$ which define the Bravais lattice type, see Table 1 and Sec. 2.2. Further, both moiré lattice vectors $\underline{R}_{M 1}, \underline{R}_{M 2}$ are scaled and rotated by the same amount with respect to their counterparts $\underline{\mathrm{R}}_{01}, \underline{\mathrm{R}}_{\mathrm{o} 2}$ of the substrate lattice. This shows that for isotropically scaled and rotated overlayers the Bravais lattice type of a possible moire lattice is always that of the initial substrate lattice. Thus, Figs. 8, 9 are of general validity. As an illustration, Fig. 10 shows moiré patterns for fictitious surfaces of different Bravais lattices where the patterns result from rotation by $\alpha=4^{\circ}$ and isotropic scaling by $\mathrm{p}=0.95$. This corresponds in all examples to a moiré angle $\gamma=55.71^{\circ}$ and a moiré factor $\kappa=11.252$. Obviously, for all four Bravais lattice types with symmetry, primitive and centered rectangular, square, and hexagonal, the type of the moiré lattice agrees with that of the substrate and overlayer lattice. While this theoretical result is quite clear its experimental verification seems to exist only for hexagonal surface systems so far.

Relations (53), (54), (55) can be inverted to yield rotation angles $\alpha$ and scaling factors $p$ as functions of the moiré angle $\gamma$ and moiré factor $\kappa$ according to

$$
\begin{aligned}
& \cos (\alpha)=\frac{\cos (\gamma)+\kappa}{\sqrt{1+\kappa^{2}+2 \kappa \cos (\gamma)}}, \quad \tan (\alpha)=\frac{\sin (\gamma)}{[\cos (\gamma)+\kappa]} \\
& \mathrm{p}=\frac{\kappa}{\sqrt{1+\kappa^{2}+2 \kappa \cos (\gamma)}} .
\end{aligned}
$$


This can be particularly useful when rotation and scaling needs to be quantified from experimentallly identified moiré patterns.

\subsection{General ( $\left.p_{1} \times p_{2}\right) R \alpha$ overlayers}

Moiré patterns of general rotated and anisotropically scaled $\left(\mathrm{p}_{1} \times \mathrm{p}_{2}\right) \mathrm{R} \alpha$ overlayers seem to have been explored by experiment only in very few special cases. As an example, Fig. 11 shows a simulation of results from early low energy electron diffraction (LEED) studies of the reconstructed quasihexagonal gold surface described as $\mathrm{Au}(111)-(\sqrt{3} \times 22)$ rect [31]. Here the unilateral compression of the overlayer results in a striped moiré pattern as discussed below. (Recent STM studies [32] indicate a more complex herringbone structure with striped domains.)

Moiré interference of rotated and anisotropically scaled $\left(\mathrm{p}_{1} \times \mathrm{p}_{2}\right) \mathrm{R} \alpha$ overlayers has been decribed in detail in Sec. 2.2 where the transformation between overlayer and substrate lattice vectors of general Bravais lattices is given by (11). The resulting moiré lattice vectors $\underline{\mathrm{R}}_{\mathrm{M} 1}, \underline{\mathrm{R}}_{\mathrm{M} 2}$ are determined by (12), (13) while the moiré factors $\kappa_{1}, \kappa_{2}$ and moiré angles $\gamma_{1}, \gamma_{2}$ are described by relations (14), (15), (16), (17), respectively. Here the Bravais lattice type of the moiré lattice will, in general, not agree with that of the surface lattice which becomes evident from the analytical formulas. As an illustration Fig. 12 shows the moiré pattern of a fictitious hexagonal overlayer on hexagonal substrate described as $(0.940 \times 0.983) \mathrm{R}^{\circ}$ where the the moiré lattice describing ellipsoidal moirons is almost rectangular.

Moirons at $\left(\mathrm{p}_{1} \times \mathrm{p}_{2}\right) \mathrm{R} \alpha$ overlayers will, in general, be distorted anisotropically and even more complex details such as stripes, spirals, or zig-zag patterns can appear. Here the special case of anisotropically scaled overlayers without rotation, $\left(\mathrm{p}_{1} \times \mathrm{p}_{2}\right)$, is interesting. Setting $\alpha=0^{\circ}$ in the general relations (11), (12), (13), (14), (15), (16), (17) yields for the transformation between overlayer and substrate lattice vectors

$$
\left(\begin{array}{l}
\underline{\mathrm{R}}_{\mathrm{o} 1}{ }^{\prime} \\
\underline{\mathrm{R}}_{\mathrm{o} 2}{ }^{\prime}
\end{array}\right)=\left(\begin{array}{cc}
\mathrm{p}_{1} & 0 \\
0 & \mathrm{p}_{2}
\end{array}\right) \cdot\left(\begin{array}{l}
\underline{\mathrm{R}}_{\mathrm{o} 1} \\
\underline{\mathrm{R}}_{\mathrm{o} 2}
\end{array}\right),
$$

and for the moiré lattice vectors $\underline{\mathrm{R}}_{\mathrm{M} 1}, \underline{\mathrm{R}}_{\mathrm{M} 2}$ 


$$
\left(\begin{array}{l}
\underline{\mathrm{R}}_{\mathrm{M} 1} \\
\underline{\mathrm{R}}_{\mathrm{M} 2}
\end{array}\right)=\left(\begin{array}{cc}
\frac{\mathrm{p}_{1}}{1-\mathrm{p}_{1}} & 0 \\
0 & \frac{\mathrm{p}_{2}}{1-\mathrm{p}_{2}}
\end{array}\right) \cdot\left(\begin{array}{l}
\underline{\mathrm{R}}_{\mathrm{o} 1} \\
\underline{\mathrm{R}}_{\mathrm{o} 2}
\end{array}\right)
$$

leading to moiré parameters

$$
\begin{aligned}
& \kappa_{i}=\frac{R_{M i}}{R_{o i}}=\frac{p_{i}}{\left|1-p_{i}\right|} \quad i=1,2, \\
& \gamma_{1}=\left\{\begin{array}{ll}
0^{\circ} & \text { for } p_{2}<1 \\
180^{\circ} & \text { for } p_{2}>1
\end{array}, \quad \gamma_{2}=\left\{\begin{array}{ll}
0^{\circ} & \text { for } p_{1}<1 \\
180^{\circ} & \text { for } p_{1}>1
\end{array} .\right.\right.
\end{aligned}
$$

Obviously, both moiré lattice vectors $\underline{R}_{M 1}, \underline{R}_{M 2}$ can diverge separately for scaling factors approaching $\mathrm{p}_{\mathrm{i}}=1$. For $\mathrm{p}_{1}=1, \mathrm{p}_{2} \neq 1$ only moiré lattice vector $\underline{\mathrm{R}}_{\mathrm{M} 2}$ will be finite giving rise to a $1-$ dimensional periodic moiré pattern where the moirons appear as infinite stripes rather than finite regions. This reflects exactly the experimental findings of the early analysis of the reconstructed $\mathrm{Au}(111)-(\sqrt{3} \times 22)$ rect [31] surface shown in Fig. 11. Here the overlayer can be characterized as $\left(\mathrm{p}_{1}\right.$ $\left.\mathrm{x} \mathrm{p}_{2}\right) \mathrm{R} \alpha$ with $\mathrm{p}_{1}=1, \mathrm{p}_{2}=22 / 23, \alpha=0^{\circ}$ if the substrate surface is described by rectangular Bravais lattice vectors $\underline{\mathrm{R}}_{\mathrm{o}}, \underline{\mathrm{R}}_{\mathrm{o} 2}$.

\section{CONCLUSION}

The present studies provide a solid theoretical foundation which allows determining the detailed geometric behavior of possible moiré patterns resulting from periodic overlayers at single crystal surfaces described as $\left(\mathrm{p}_{1} \times \mathrm{p}_{2}\right) \mathrm{R} \alpha$ where $\alpha$ denotes overlayer rotation angles and $\mathrm{p}_{1}, \mathrm{p}_{2}$ are scaling factors. This includes surfaces of all possible Bravais types with and without lattice bases. Clearly, the present treatment does not account for details of the electronic overlayer - substrate coupling and binding within the overlayer which may be rather complex and difficult to evaluate. However, the periodicity of the moiré patterns, interpreted as a phenomenon of spatial interference between overlayer and substrate, is described for realistic surface systems rather reliably, as shown for hexagonal monolayers on hexagonal metal substrate in comparison with experiment.

The mathematical formalism based on 2-dimensional Fourier analysis provides a simple geometric interpretation of the quasiperiodic moiré lattices together with mathematical formulas (12), 
(13), (14), (15), (16), (17), (18) determining moiré lattice vectors, lattice constants, and angles for surfaces of all possible Bravais types. These formulas show, in particular, that for isotropic scaling and rotation the Bravais lattice type of the moiré lattice agrees always with that of the surface system. The theoretical treatment yields also boundaries for layer rotation angles $\alpha$ and scaling factors $\mathrm{p}_{1}, \mathrm{p}_{2}$ which result in meaningful, i.e. clearly observable moiré patterns. For isotropic scaling, reasonable factors $\mathrm{p}$ range between 0.8 and 1.2 while overlayer rotation with clear moiré patterns is limited to rotation angles $\alpha$ below $10^{\circ}$. Further, reasonable approximate relationships for the moiré lattice vectors of surfaces referring to very small overlayer rotation angles $\alpha$, corresponding to very large moiré lattice vectors, are obtained. Here the moiré lattice constants are found to scale with $1 / \alpha$.

So far, the experimental verification of the present mathematical formalism is restricted almost exclusively to hexagonal overlayers, such as graphene or h-BN, on top of hexagonal substrate, which includes (111) oriented surfaces of fcc or (0001) surfaces of hcp metal substrate as discussed in the introduction. For these systems the present theory can describe the observed moiré patterns quite accurately. However, the formation of moiré patterns can be expected to occur at general close packed surfaces and, hopefully, the present theoretical treatment will stimulate also quantitative experimental work along these lines. Further, interference phenomena involving multiple overlayer stacking may be considered by theory in an attempt to describe, for example, graphene bilayer stacking on metal surfaces. Work in this direction is presently under way.

\section{ACKNOWLEDGEMENT}

Stimulating discussions with Thomas Greber (University Zürich), Michel A. Van Hove (City University of Hong Kong), and Michael S. Altman (Hong Kong University of Science and Technology) are gratefully acknowledged. 


\section{References}

[1] P. R. Watson, M. A. Van Hove, and K. Hermann, Atlas of Surface Crystallography based on the NIST Surface Structure Database (SSD), Vol.1a, b, Monograph No. 5, Journal of Physical and Chemical Reference Data, American Chemical Society, 1994.

[2] M. A. Van Hove, K. Hermann, and P. R. Watson, Acta Cryst. B 58 (2002) 338.

[3] M. A. Van Hove, K. Hermann, and P. R. Watson in Landolt-Börnstein, New Series, Vol. III/42, Physics of Covered Solid Surfaces, Springer (2002), pp. 4.1-122.

[4] E.A. Wood, J. Appl. Phys. 35 (1964) 1306.

[5] K. Hermann, Crystallography and Surface Structure, an introduction for surface scientists and nanoscientists, Wiley-VCH, Berlin 2011“, ISBN 978-3-527-41012-5.

[6] D. Martoccia, P.R. Willmott, T. Brugger, M. Björck, S. Günther, C.M. Schlepütz, A. Cervellino, S.A. Pauli, B.D. Patterson, S. Marchini, J. Wintterlin, W. Moritz, and T. Greber, Phys. Rev. Lett. 101 (2008) 126102.

[7] W. Moritz, B. Wang, M.-L. Bocquet, T. Brugger, T. Greber, J. Wintterlin, and S. Günther, Phys. Rev. Lett. 104 (2010) 136102.

[8] D. Martoccia, M. Björck, C.M. Schlepütz, T. Brugger, S.A. Pauli, B.D. Patterson, T. Greber, and P.R. Willmott, New J. Phys. 12 (2010) 043028.

[9] B. Wang M.-L. Bocquet, S. Marchini, S. Günther and J. Wintterlin, Phys. Chem. Chem. Phys 10 (2008) 3530.

[10] M. Iannuzzi and J. Hutter, Surf. Sci. 605 (2011) 1360.

[11] D. Stradi, S. Barja, C. Díaz, M. Garnica, B. Borca, J. J. Hinarejos, D. Sánchez-Portal, M. Alcamí, A. Arnau, A. L. Vázquez de Parga, R. Miranda, and F. Martín, Phys. Rev. Lett. 106 (2011) 186102.

[12] B. Wang and M.-L. Bocquet, J. Phys. Chem. Lett. 2 (2011) 2341.

[13] M. Kuwabara, D.R. Clark, and D.A. Smith, Appl. Phys. Lett. 56 (1990) 2396.

[14] A. H. MacDonald and R. Bistritzer, Nature 474 (2011) 453.

[15] A. Luican, G. Li, A. Reina, J. Kong, R. R. Nair, K. S. Novoselov, A. K. Geim, and E.Y. Andrei, Phys. Rev. Lett. 106 (2011) 126802.

[16] T.M. Bernhardt, B. Kaiser, and K. Rademann, Surf. Sci. 408 (1998) 86.

[17] A. B. Preobrajenski, M.-L. Ng, A. S. Vinogradov, and N. Mårtensson, Phys. Rev. B 78 (2008) 073401. 
[18] A.T. N'Diaye, J. Coraux, Tim N Plasa, C. Busse, and T. Michely, New J. Phys. 10 (2008) 043033.

[19] J. Coraux, A.T. N'Diaye, C. Busse, and T. Michely, Nano Lett. 8 (2008) 565.

[20] T.A. Land, T. Michely, R.J. Behm, J.C. Hemminger, and G. Comsa, Surf. Sci. 264 (1992) 261.

[21] L. Gao, J.R. Guest, and N.P. Guisinger, Nano Lett. 10 (2010) 3512.

[22] T. Brugger, S. Günther, B. Wang, J. H. Dil, M.-L. Bocquet, J. Osterwalder, J. Wintterlin, and T. Greber, Phys. Rev. B 79 (2009) 045407.

[23] M. Morscher, M. Corso, T. Greber, and J. Osterwalder, Surf. Sci. 600 (2006) 3280.

[24] M. Corso, W. Auwärter,M. Muntwiler, A. Tamai, T. Greber and J. Osterwalder, Science 303 (2004) 217.

[25] D. Martoccia, S.A. Pauli, T. Brugger, T. Greber, B.D. Patterson, and P.R. Willmott, Surf. Sci. 604 (2010) L9.

[26] C. Chambon, A. Coati, and Y. Garreau, Surf. Sci. 602 (2008) 2363.

[27] C. Chambon, J. Creuze, A. Coati, M. Sauvage-Simkin, and Y. Garreau, Phys. Rev. B 79 (2009) 125412.

[28] G. Lebanon and A. M. Bruckstein in Energy Minimization Methods in Computer Vision and Pattern Recognition, A. K. Jain, M. Figueiredo, and J. Zerubia, Springer Heidelberg, 2001, p. 185.

[29] W. Zhao, S.M. Kozlov, Ol. Höfert, K. Gotterbarm, M.P.A. Lorenz, F. Viñes, C. Papp, A. Görling, H.-P. Steinrück, J. Phys. Chem. Lett. 2 (2011) 759.

[30] J. Xue, J. Sanchez-Yamagishi, D. Bulmash, P. Jacquod, A. Deshpande, K. Watanabe, T. Taniguchi, P. Jarillo-Herrero, and B.J. LeRoy, Nature Mat. 10 (2011) 282.

[31] M.A. Van Hove, R.J. Koestner, P.C. Stair, J.P. Bibérian, L.L. Kesmodel, I. Bartoš, and G.A. Somorjai, Surf. Sci. 103 (1981) 189.

[32] L. Tang, X. Zhang, Q. Guo, Y. Wu, L. Wang, and H. Cheng, Phys. Rev. B 82 (2010) 125414. 


\section{Tables}

Table 1. Lattice vectors $\underline{\mathrm{R}}_{01}, \underline{\mathrm{R}}_{02}$ of the five 2-dimensional Bravais lattices. The corresponding vector lengths ratios $q$ are defined by $q=R_{01} / R_{02}$ and $\omega$ denotes the angle between $\underline{R}_{01}$ and $\underline{\mathrm{R}}_{\mathrm{o} 2}$.

\begin{tabular}{|c|c|c|}
\hline & $\mathrm{q}$ & $\omega\left[^{\circ}\right]$ \\
\hline Oblique & $\mathrm{q} \neq 1$ & $\neq 60, \neq 90, \neq 120$ \\
$\mathrm{p}$-Rectangular & $\mathrm{q} \neq 1$ & 90 \\
$\mathrm{c}-$ Rectangular & 1 & $\neq 60, \neq 90, \neq 120$ \\
Square & 1 & 60,120 \\
Hexagonal & 1 & 60 \\
\hline
\end{tabular}

Table 2. Moiré factors $\kappa$ for graphene forming ( $\mathrm{p} x \mathrm{p}$ ) overlayers on different metal substrate. Scaling factors $\mathrm{p}$ are derived from ratios of corresponding lattice constants while moiré factors $\kappa$ are determined using relation (34). The references denote experimental studies where the moiré patterns have been identified.

\begin{tabular}{|l|c|c|}
\hline \multicolumn{1}{|c|}{ Substrate } & $\mathrm{p}$ & $\kappa$ \\
\hline $\operatorname{Pt}(111)[17,20]$ & 0.89 & 8.09 \\
$\operatorname{Ir}(111)[18,19]$ & 0.91 & 10.11 \\
$\operatorname{Ru}(0001)[6-12]$ & 0.92 & 11.50 \\
$\operatorname{Rh}(111)[12,17]$ & 0.93 & 13.28 \\
$\mathrm{Cu}(111)[21]$ & 0.97 & 32.33 \\
$\operatorname{Ni}(111)[29]$ & 1.00 & $\infty$ \\
\hline
\end{tabular}




\section{Figure captions}

Fig. 1. Graphene overlayer adsorbed at the $\mathrm{Ru}(0001)$ surface [7]. The Ru substrate surface is shown by its topmost three layers. (a) View perpendicular to the surface. The periodicity of the superlattice is indicated by arrows referring to moiré lattice vectors. (b) View almost parallel to the surface illustrating the periodic overlayer warping connected with the moiré patterns shown in (a).

Fig. 2. Definition of lattice vectors and angles of the substrate, $\underline{\mathrm{R}}_{01}, \underline{\mathrm{R}}_{\mathrm{o} 2}, \omega$, of the overlayer, $\underline{\mathrm{R}}_{\mathrm{o1}}, \underline{\mathrm{R}}_{\mathrm{O} 2}$, and of the moiré lattice, $\underline{\mathrm{R}}_{\mathrm{M} 1}, \underline{\mathrm{R}}_{\mathrm{M} 2}, \gamma_{1}, \gamma_{2}$.

Fig. 3. Moiré pattern of a graphene overlayer at the Pt(111) surface [20] given by a balls-andsticks simulation for a view perpendicular to the surface, see text. Moiré vectors $\underline{\mathrm{R}}_{M 1}$, $\underline{\mathrm{R}}_{\mathrm{M} 2}$ are shown by arrows and labeled accordingly.

Fig. 4. Moiré pattern of a graphene layer rotated by $\alpha=3.5^{\circ}$ (indicated by lines at the bottom) [16] with respect to underlying graphene. Moiré vectors $\underline{R}_{M 1}, \underline{R}_{M 2}$ are shown by arrows and labeled accordingly. The lattice vectors $\underline{\mathrm{R}}_{01}, \underline{\mathrm{R}}_{02}$ of the underlying graphene monolayer are sketched at the lower left where a magnification by a factor 5 is applied for better visibility.

Fig. 5. Moiré pattern of a graphene layer rotated by $\alpha=3.5^{\circ}$ (indicated by lines at the bottom) with respect to underlying graphene for an overlayer shift vector of (a) $\underline{v}=-1 / 3 \underline{R}_{02}$, (b) $\underline{v}=-2 / 3 \underline{R}_{02}$, see text. (The pattern for $\underline{v}=\underline{0}$ is shown as Fig. 4.) Moiré vectors $\underline{R}_{M 1}, \underline{R}_{M 2}$ and the moiré shift $\underline{\mathrm{V}}$ are shown by arrows and labeled accordingly. The lattice vectors $\underline{\mathrm{R}}_{\mathrm{o1}}, \underline{\mathrm{R}}_{\mathrm{o} 2}$ of the underlying graphene monolayer and shift vector $\underline{\mathrm{v}}$ are sketched at the lower left where a magnification by a factor 5 is applied for better visibility.

Fig. 6. Moiré pattern of a graphene layer near the edge of a monoatomic step at the $\operatorname{Ir}(111)$ surface, see text. The step edge separating adjacent terraces is emphasized by a dashed line. Moiré vectors $\underline{\mathrm{R}}_{\mathrm{M} 1}, \underline{\mathrm{R}}_{\mathrm{M} 2}$ and the moiré shift $\underline{\mathrm{V}}$ are shown by arrows and labeled accordingly. Lattice vectors $\underline{\mathrm{R}}_{01}, \underline{\mathrm{R}}_{\mathrm{o} 2}$ at the terraces and the effective lateral shift $-\underline{\mathrm{v}}$ of Ir substrate atoms is shown at the bottom where a magnification by a factor 3.5 is applied for better visibility.

Fig. 7. Moiré pattern of a graphene overlayer on top of a hexagonal boron nitride (h-BN) layer corresponding to lattice constants differing by $2 \%(\mathrm{p}=0.98)$ and a rotation angle of $\alpha=$ $5.4^{\circ}$ (indicated by lines at the bottom) [30]. Moiré vectors $\underline{\mathrm{R}}_{\mathrm{M} 1}, \underline{\mathrm{R}}_{\mathrm{M} 2}$ are shown by arrows and labeled accordingly. The lattice vectors $\underline{\mathrm{R}}_{01}, \underline{\mathrm{R}}_{02}$ of the underlying $\mathrm{h}-\mathrm{BN}$ layer are shown at the lower left where a magnification by a factor 5 is applied for better visibility.

Fig. 8. Perspective view of moiré angles $\gamma(\mathrm{p}, \alpha)$ according to (55) for rotation angles $0 \leq \alpha \leq$ $10^{\circ}$ and isotropic scaling factors $0.9 \leq \mathrm{p} \leq 1.1$, see text. 
Fig. 9. Perspective view of moiré factors $\kappa(p, \alpha)$ according to (53) for rotation angles $0 \leq \alpha \leq$ $10^{\circ}$ and isotropic scaling factors $0.9 \leq \mathrm{p} \leq 1.1$, see text.

Fig. 10. Moiré patterns of surface systems with different Bravais lattices, (a) primitive rectangular (p-rect., $\mathrm{R}_{\mathrm{o} 1} / \mathrm{R}_{\mathrm{o} 2}=1.3$ ), (b) centered rectangular (c-rect., $\left.\omega=\angle\left(\underline{\mathrm{R}}_{01}, \underline{\mathrm{R}}_{02}\right)=75^{\circ}\right)$, (c) square, (d) hexagonal (hex.). The patterns result from overlayer rotation by $\alpha=4^{\circ}$ (indicated by lines at the bottom) and isotropic scaling by $\mathrm{p}=0.95$. Moiré vectors $\underline{\mathrm{R}}_{\mathrm{M} 1}$, $\underline{\mathrm{R}}_{\mathrm{M} 2}$ are shown inside their unit cells by arrows and labeled accordingly. Unit cells and lattice vectors $\underline{\mathrm{R}}_{01}, \underline{\mathrm{R}}_{02}$ of the substrate (unlabeled arrows) are included at the lower left where a magnification by a factor 3 is applied for better visibility.

Fig. 11. Moiré pattern of a unilaterally compressed hexagonal overlayer on hexagonal substrate referring to structure parameters $\mathrm{p}_{1}=1, \mathrm{p}_{2}=22 / 23, \alpha=0^{\circ}$ using rectangular Bravais lattice vectors $\underline{\mathrm{R}}_{01}, \underline{\mathrm{R}}_{02}$, see text. Lattice vectors $\underline{\mathrm{R}}_{01}, \underline{\mathrm{R}}_{\mathrm{0} 2}$ and the moiré lattice vector $\underline{\mathrm{R}}_{\mathrm{M} 2}=$ $22 \underline{\mathrm{R}}_{02}$ are sketched and labeled accordingly. The geometry has been suggested in early LEED studies of the reconstructed $\mathrm{Au}(111)-(\sqrt{3} \times 22)$ rect surface [31].

Fig. 12. Moiré pattern of a fictitious hexagonal overlayer on hexagonal substrate described as $(0.940 \times 0.983) \mathrm{R} 5^{\circ}$. Moiré vectors $\underline{\mathrm{R}}_{\mathrm{M} 1}, \underline{\mathrm{R}}_{\mathrm{M} 2}$ are shown by arrows and labeled accordingly. The periodicity of the substrate layer is illustrated by removing the overlayer at the upper right of the section. 
(a)
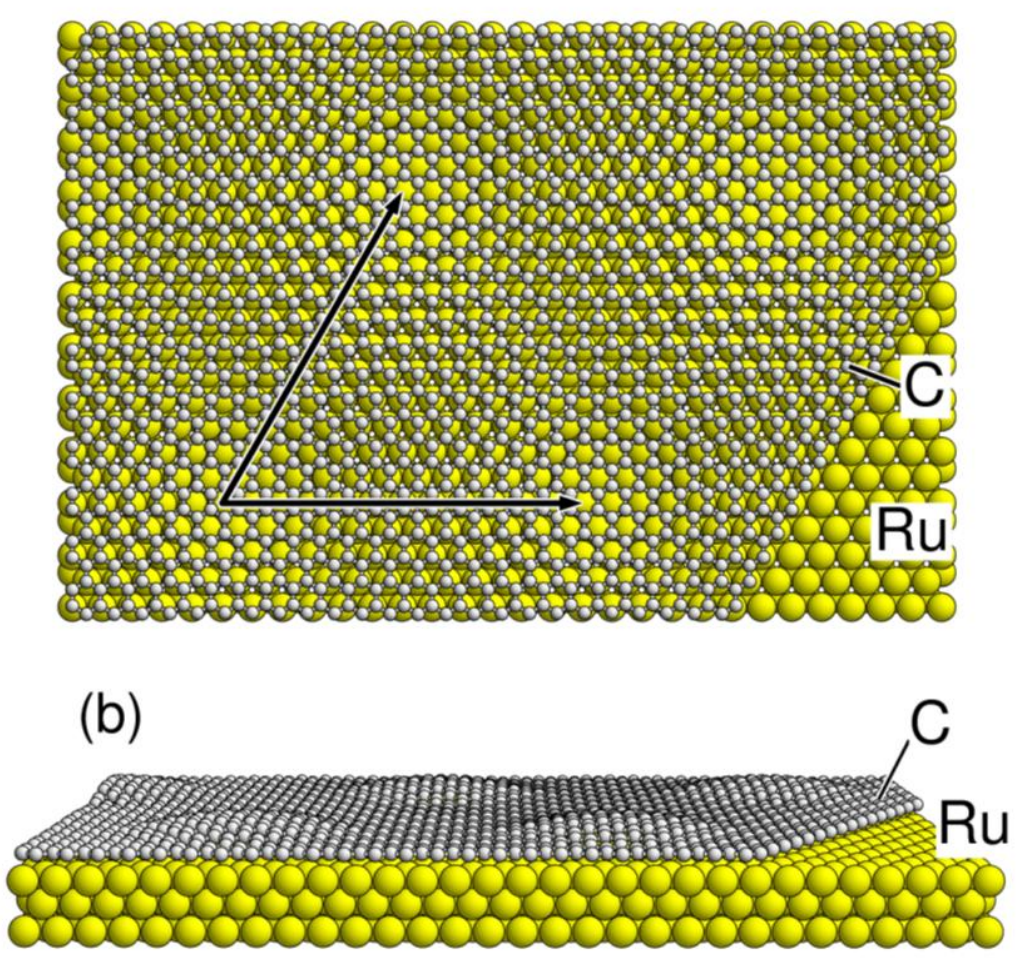

Fig. 1. Graphene overlayer adsorbed at the $\mathrm{Ru}(0001)$ surface [7]. The $\mathrm{Ru}$ substrate surface is shown by its topmost three layers. (a) View perpendicular to the surface. The periodicity of the superlattice is indicated by arrows referring to moiré lattice vectors. (b) View almost parallel to the surface illustrating the periodic overlayer warping connected with the moiré patterns shown in (a). 


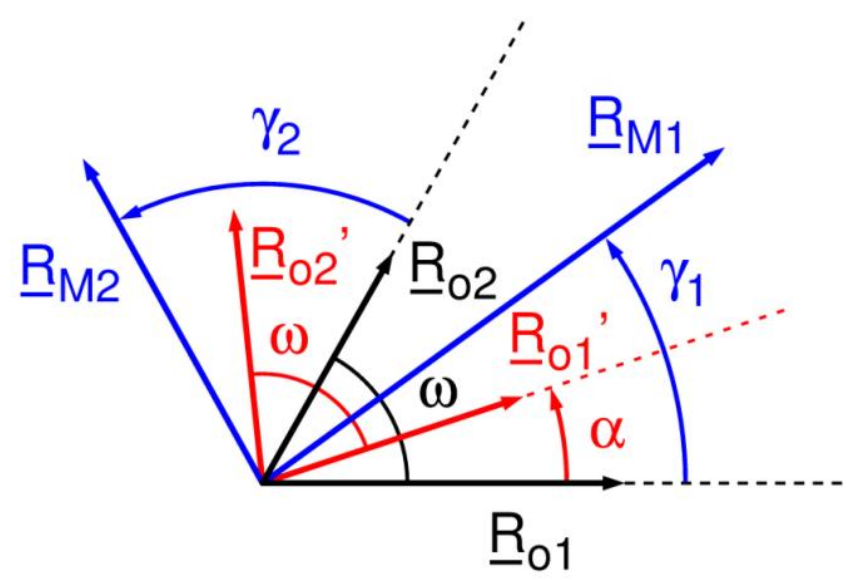

Fig. 2. Definition of lattice vectors and angles of the substrate, $\underline{\mathrm{R}}_{01}, \underline{\mathrm{R}}_{02}$, $\omega$, of the overlayer, $\underline{\mathrm{R}}_{\mathrm{o} 1}, \underline{\mathrm{R}}_{\mathrm{O} 2}$, and of the moiré lattice, $\underline{\mathrm{R}}_{\mathrm{M} 1}, \underline{\mathrm{R}}_{\mathrm{M} 2}, \gamma_{1}, \gamma_{2}$. 


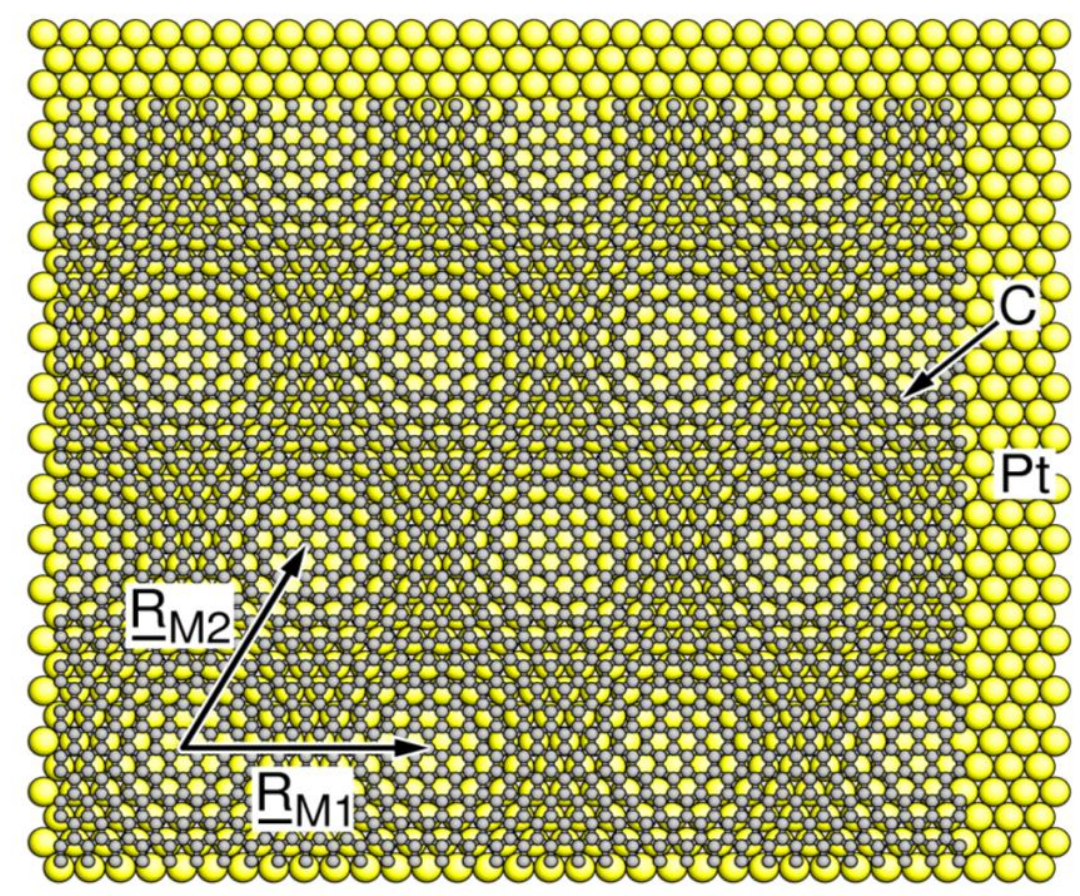

Fig. 3. Moiré pattern of a graphene overlayer at the $\operatorname{Pt}(111)$ surface [20] given by a balls-andsticks simulation for a view perpendicular to the surface, see text. Moiré vectors $\underline{R}_{\mathrm{M} 1}$, $\underline{\mathrm{R}}_{\mathrm{M} 2}$ are shown by arrows and labeled accordingly. 


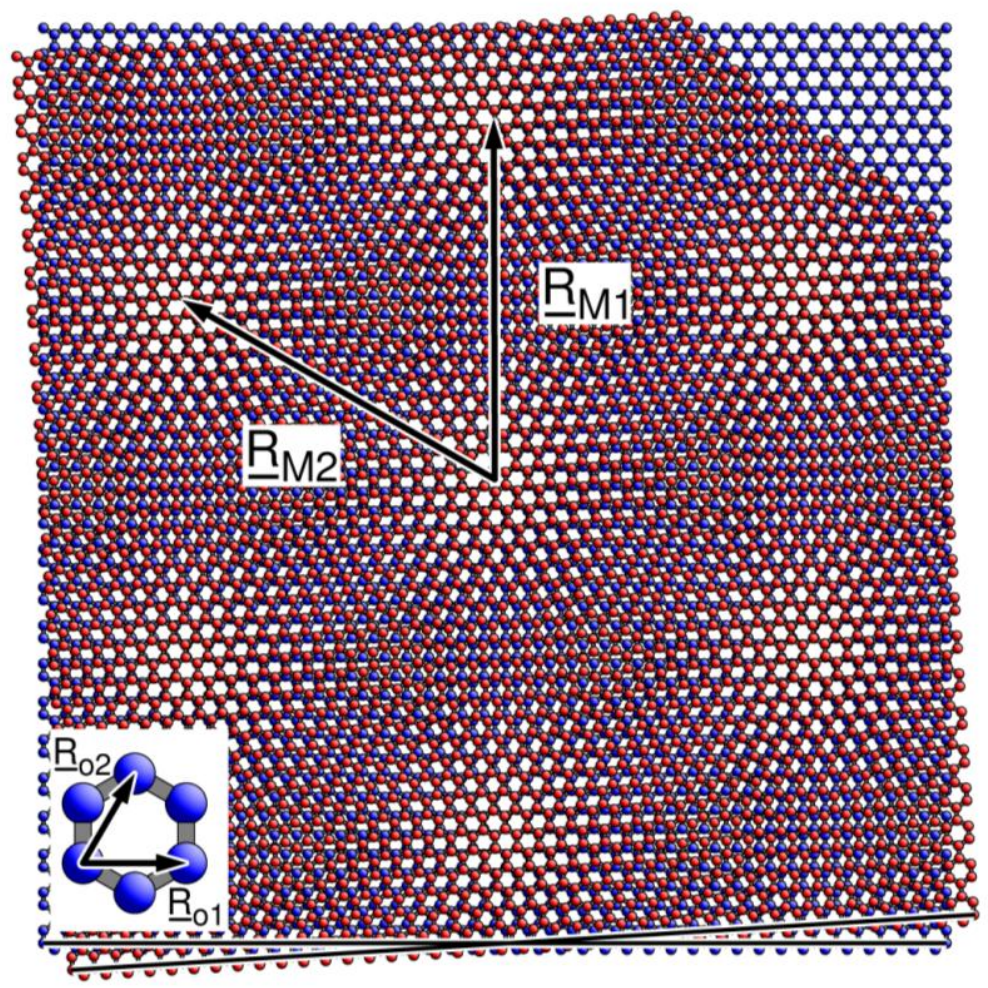

Fig. 4. Moiré pattern of a graphene layer rotated by $\alpha=3.5^{\circ}$ (indicated by lines at the bottom) [16] with respect to underlying graphene. Moiré vectors $\underline{\mathrm{R}}_{\mathrm{M} 1}, \underline{\mathrm{R}}_{\mathrm{M} 2}$ are shown by arrows and labeled accordingly. The lattice vectors $\underline{\mathrm{R}}_{\mathrm{o} 1}, \underline{\mathrm{R}}_{\mathrm{0} 2}$ of the underlying graphene monolayer are sketched at the lower left where a magnification by a factor 5 is applied for better visibility. 
(a)

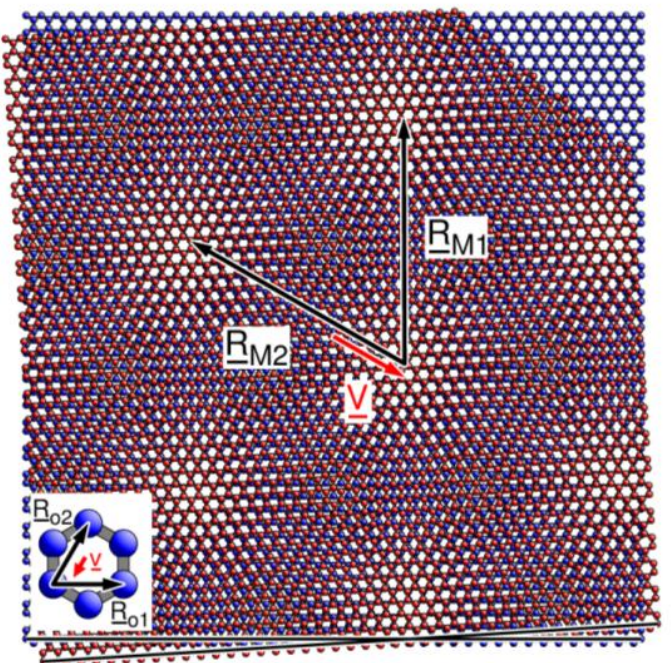

(b)

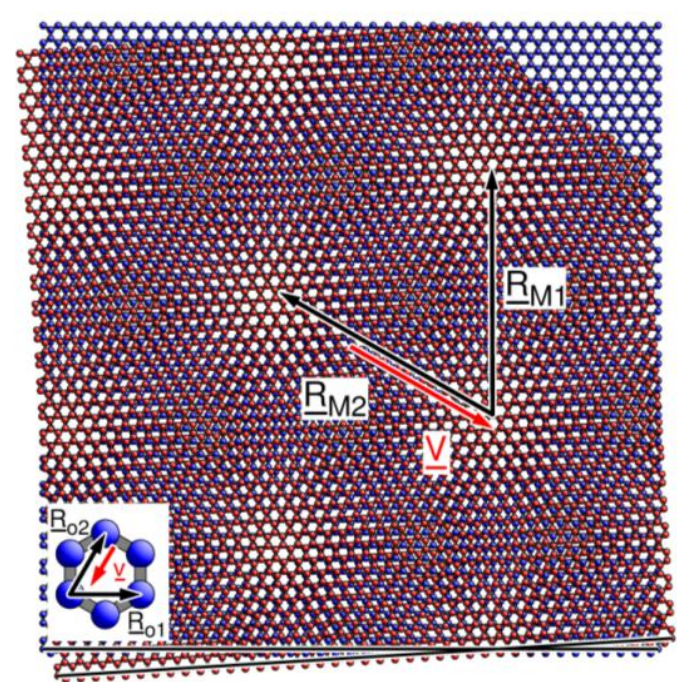

Fig. 5. Moiré pattern of a graphene layer rotated by $\alpha=3.5^{\circ}$ (indicated by lines at the bottom) with respect to underlying graphene for an overlayer shift vector of (a) $\underline{v}=-1 / 3 \underline{R}_{02}$, (b) $\underline{v}=-2 / 3 \underline{R}_{02}$, see text. (The pattern for $\underline{v}=\underline{0}$ is shown as Fig. 4.) Moiré vectors $\underline{R}_{M 1}, \underline{R}_{M 2}$ and the moiré shift $\underline{\mathrm{V}}$ are shown by arrows and labeled accordingly. The lattice vectors $\underline{\mathrm{R}}_{\mathrm{o} 1}, \underline{\mathrm{R}}_{\mathrm{o} 2}$ of the underlying graphene monolayer and shift vector $\underline{\mathrm{v}}$ are sketched at the lower left where a magnification by a factor 5 is applied for better visibility. 


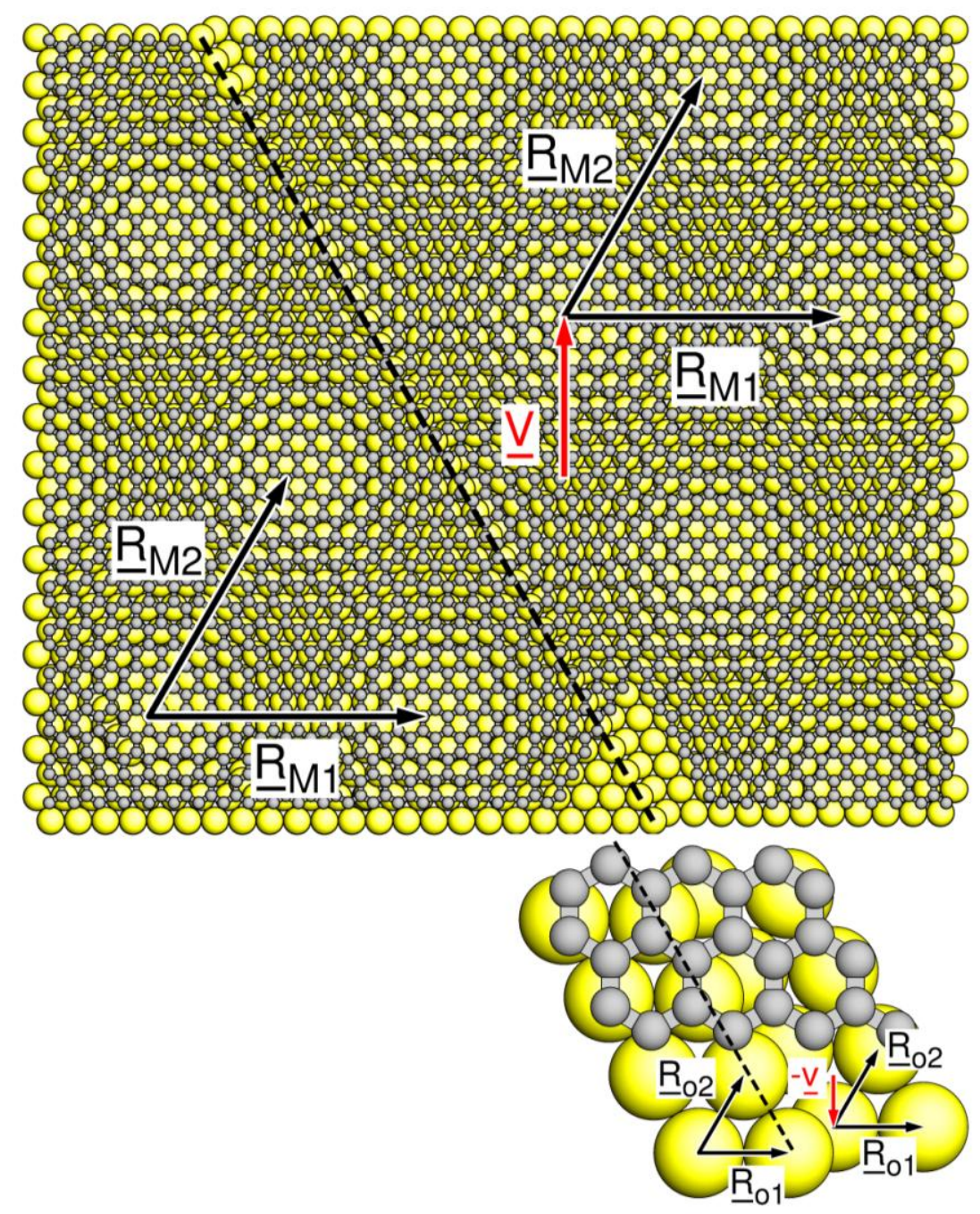

Fig. 6. Moiré pattern of a graphene layer near the edge of a monoatomic step at the $\operatorname{Ir}(111)$ surface, see text. The step edge separating adjacent terraces is emphasized by a dashed line. Moiré vectors $\underline{\mathrm{R}}_{\mathrm{M} 1}, \underline{\mathrm{R}}_{\mathrm{M} 2}$ and the moiré shift $\underline{\mathrm{V}}$ are shown by arrows and labeled accordingly. Lattice vectors $\underline{\mathrm{R}}_{\mathrm{o} 1}, \underline{\mathrm{R}}_{\mathrm{o} 2}$ at the terraces and the effective lateral shift $-\underline{\mathrm{v}}$ of Ir substrate atoms is shown at the bottom where a magnification by a factor 3.5 is applied for better visibility. 


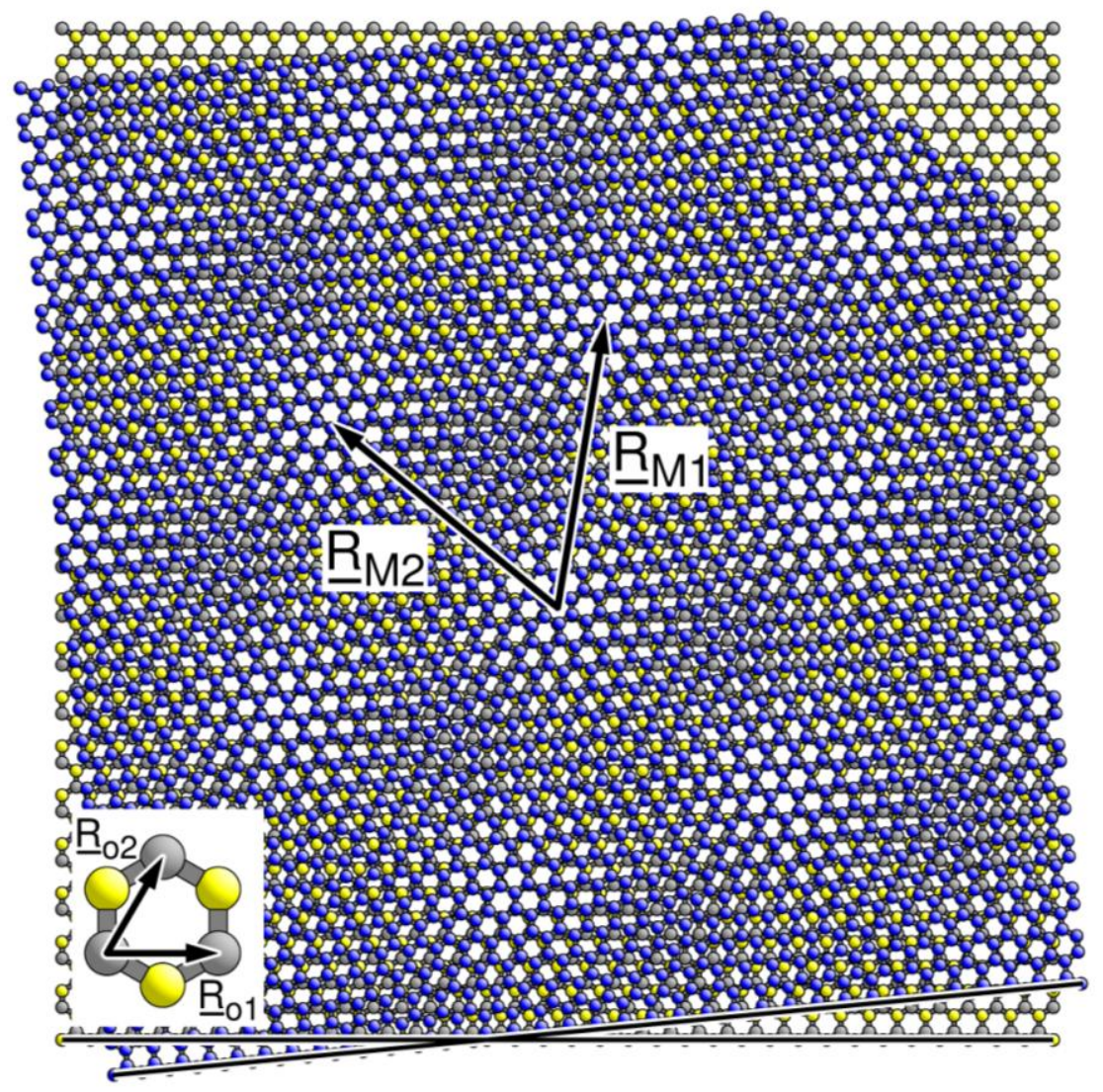

Fig. 7. Moiré pattern of a graphene overlayer on top of a hexagonal boron nitride (h-BN) layer corresponding to lattice constants differing by $2 \%(\mathrm{p}=0.98)$ and a rotation angle of $\alpha=$ $5.4^{\circ}$ (indicated by lines at the bottom) [30]. Moiré vectors $\underline{\mathrm{R}}_{\mathrm{M} 1}, \underline{\mathrm{R}}_{\mathrm{M} 2}$ are shown by arrows and labeled accordingly. The lattice vectors $\underline{\mathrm{R}}_{01}, \underline{\mathrm{R}}_{02}$ of the underlying $\mathrm{h}-\mathrm{BN}$ layer are shown at the lower left where a magnification by a factor 5 is applied for better visibility. 


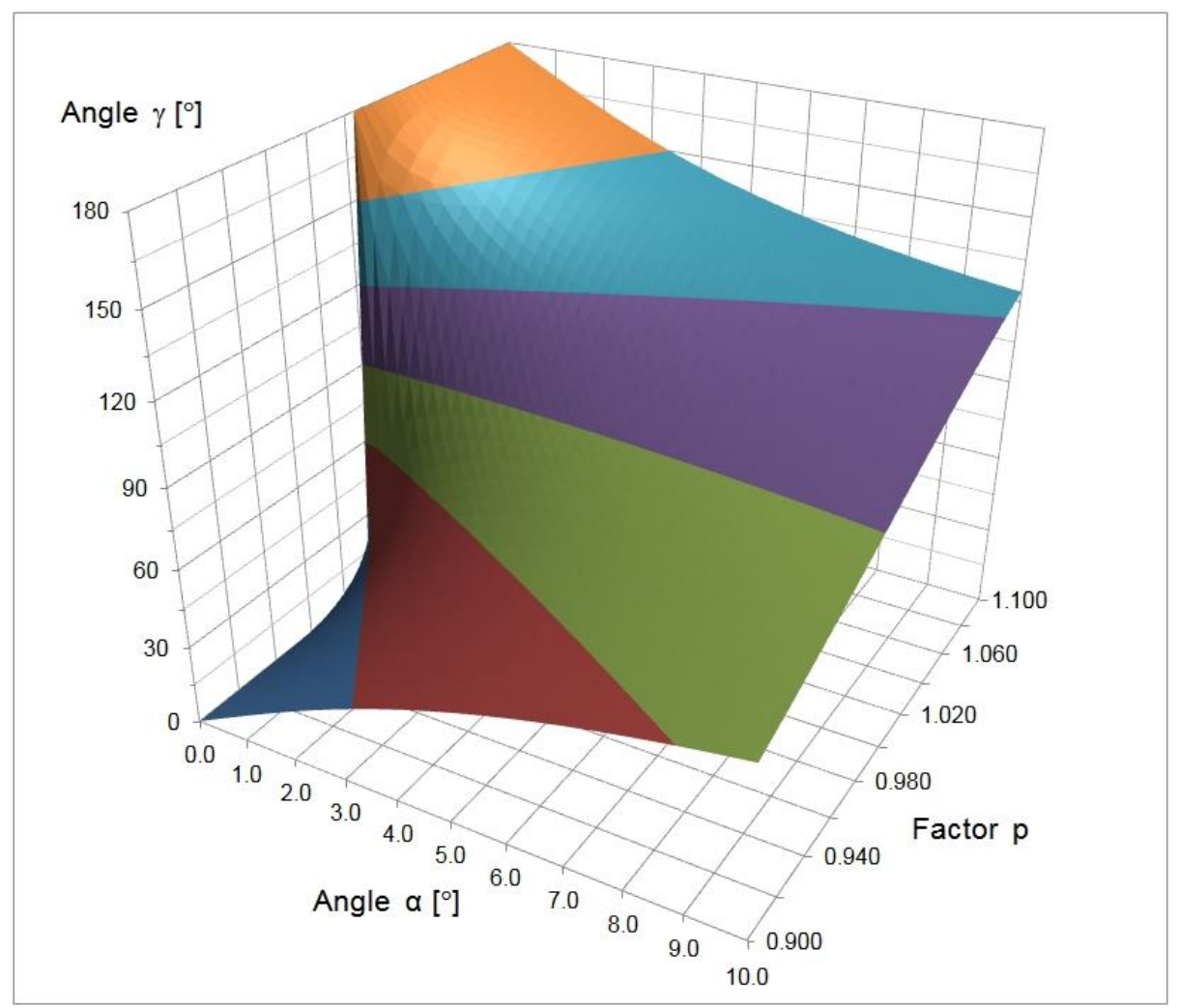

Fig. 8. Perspective view of moiré angles $\gamma(\mathrm{p}, \alpha)$ according to (55) for rotation angles $0 \leq \alpha \leq$ $10^{\circ}$ and isotropic scaling factors $0.9 \leq \mathrm{p} \leq 1.1$, see text. 


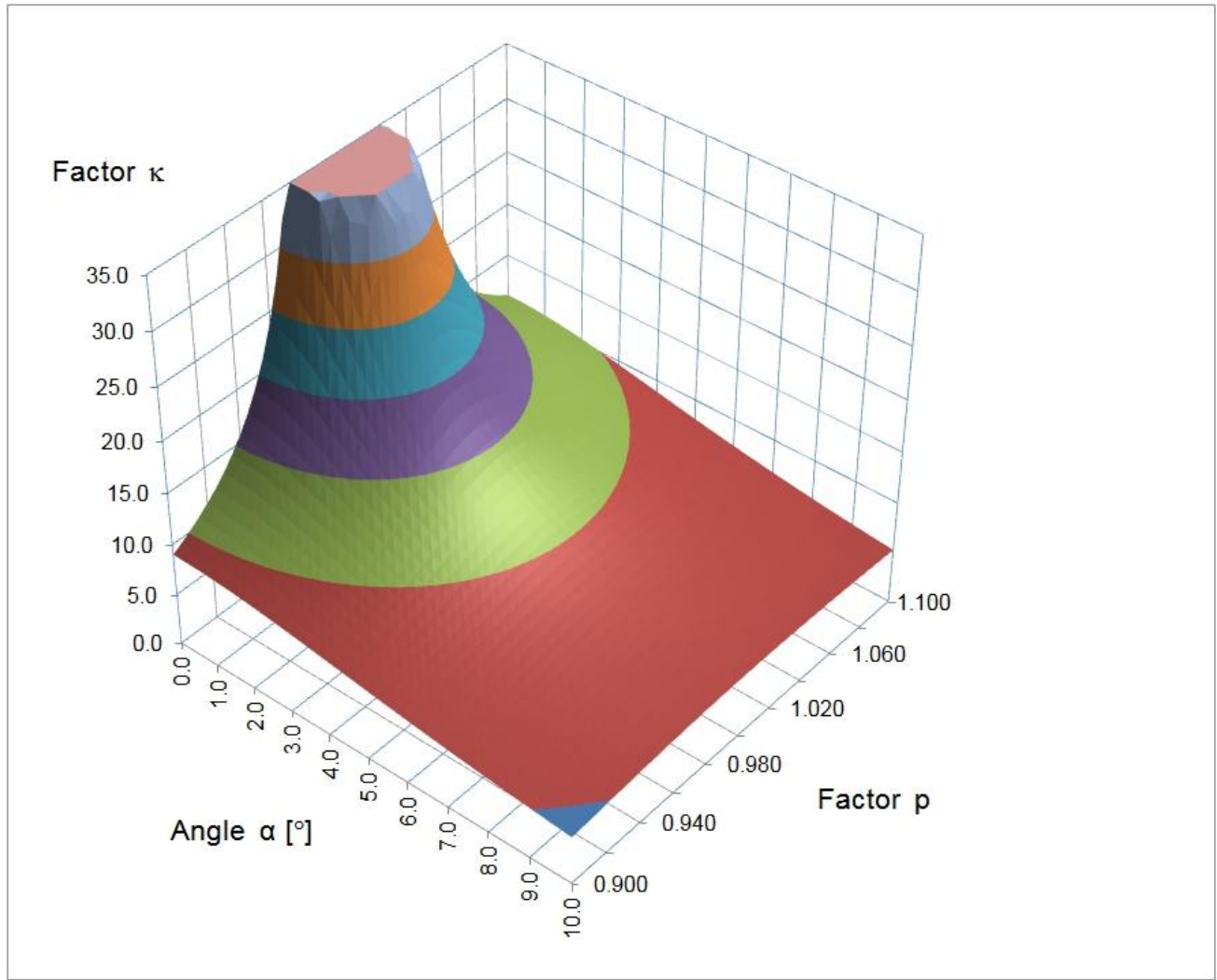

Fig. 9. Perspective view of moiré factors $\kappa(\mathrm{p}, \alpha)$ according to (53) for rotation angles $0 \leq \alpha \leq$ $10^{\circ}$ and isotropic scaling factors $0.9 \leq \mathrm{p} \leq 1.1$, see text. 
(a) p-rect.

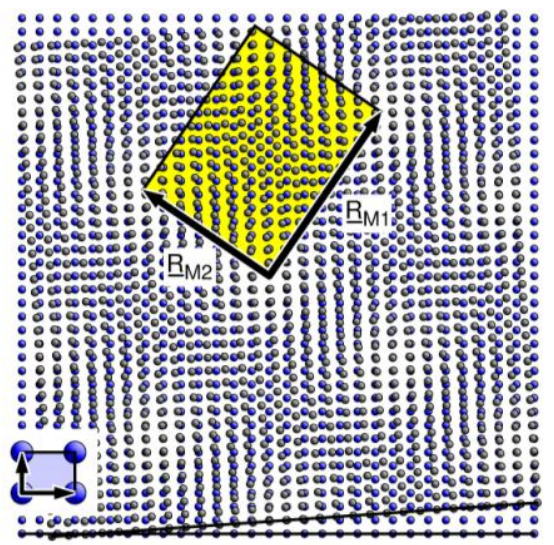

(c) square

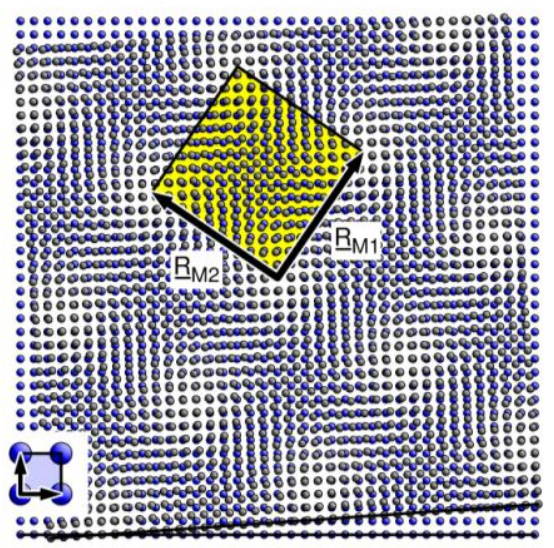

(b) c-rect.

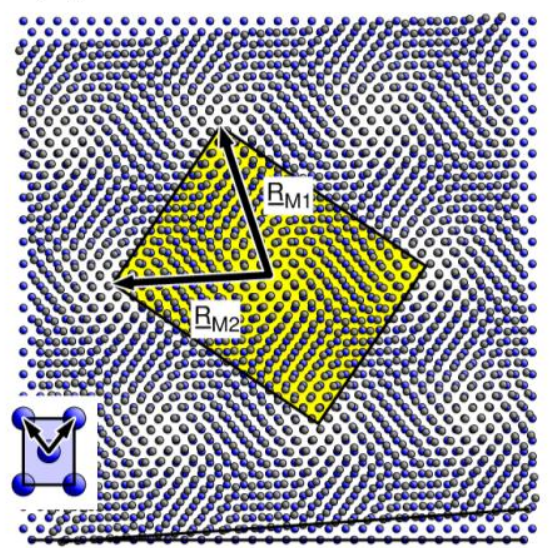

(d) hex.

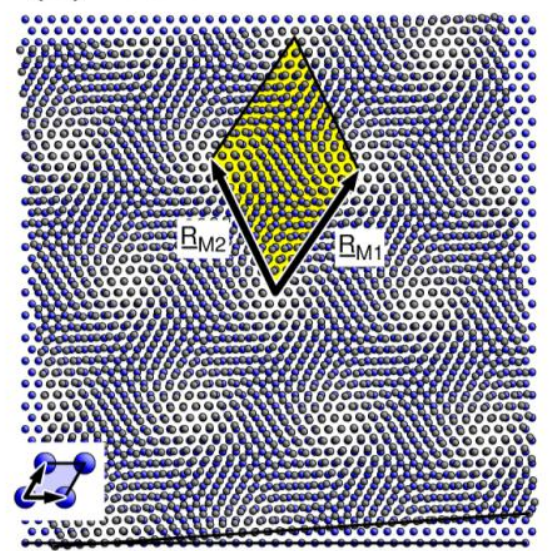

Fig. 10. Moiré patterns of surface systems with different Bravais lattices, (a) primitive rectangular (p-rect., $\mathrm{R}_{\mathrm{o} 1} / \mathrm{R}_{\mathrm{o} 2}=1.3$ ), (b) centered rectangular (c-rect., $\omega=\angle\left(\underline{\mathrm{R}}_{01}, \underline{\mathrm{R}}_{02}\right)=75^{\circ}$ ), (c) square, (d) hexagonal (hex.). The patterns result from overlayer rotation by $\alpha=4^{\circ}$ (indicated by lines at the bottom) and isotropic scaling by $\mathrm{p}=0.95$. Moiré vectors $\underline{\mathrm{R}}_{\mathrm{M} 1}$, $\underline{\mathrm{R}}_{\mathrm{M} 2}$ are shown inside their unit cells by arrows and labeled accordingly. Unit cells and lattice vectors $\underline{\mathrm{R}}_{01}, \underline{\mathrm{R}}_{02}$ of the substrate (unlabeled arrows) are included at the lower left where a magnification by a factor 3 is applied for better visibility. 


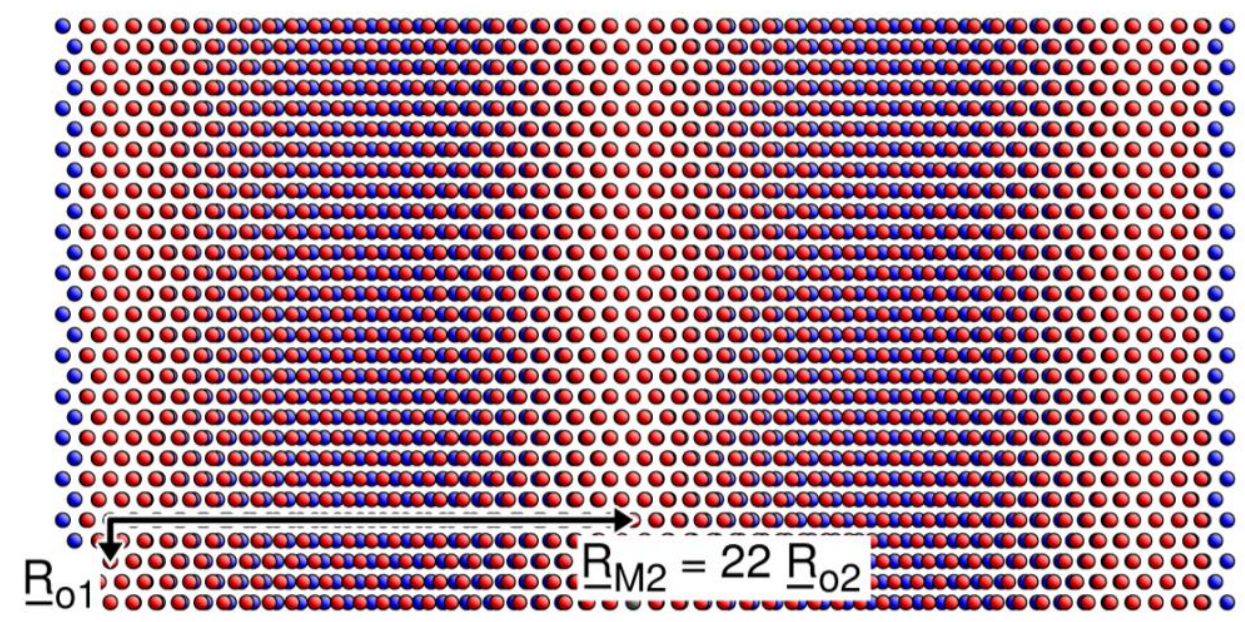

Fig. 11. Moiré pattern of a unilaterally compressed hexagonal overlayer on hexagonal substrate referring to structure parameters $\mathrm{p}_{1}=1, \mathrm{p}_{2}=22 / 23, \alpha=0^{\circ}$ using rectangular Bravais lattice vectors $\underline{R}_{01}, \underline{R}_{02}$, see text. Lattice vectors $\underline{R}_{01}, \underline{R}_{02}$ and the moiré lattice vector $\underline{R}_{M 2}=$ $22 \underline{\mathrm{R}}_{\mathrm{o} 2}$ are sketched and labeled accordingly. The geometry has been suggested in early LEED studies of the reconstructed $\mathrm{Au}(111)-(\sqrt{ } 3 \times$ 22)rect surface [31]. 


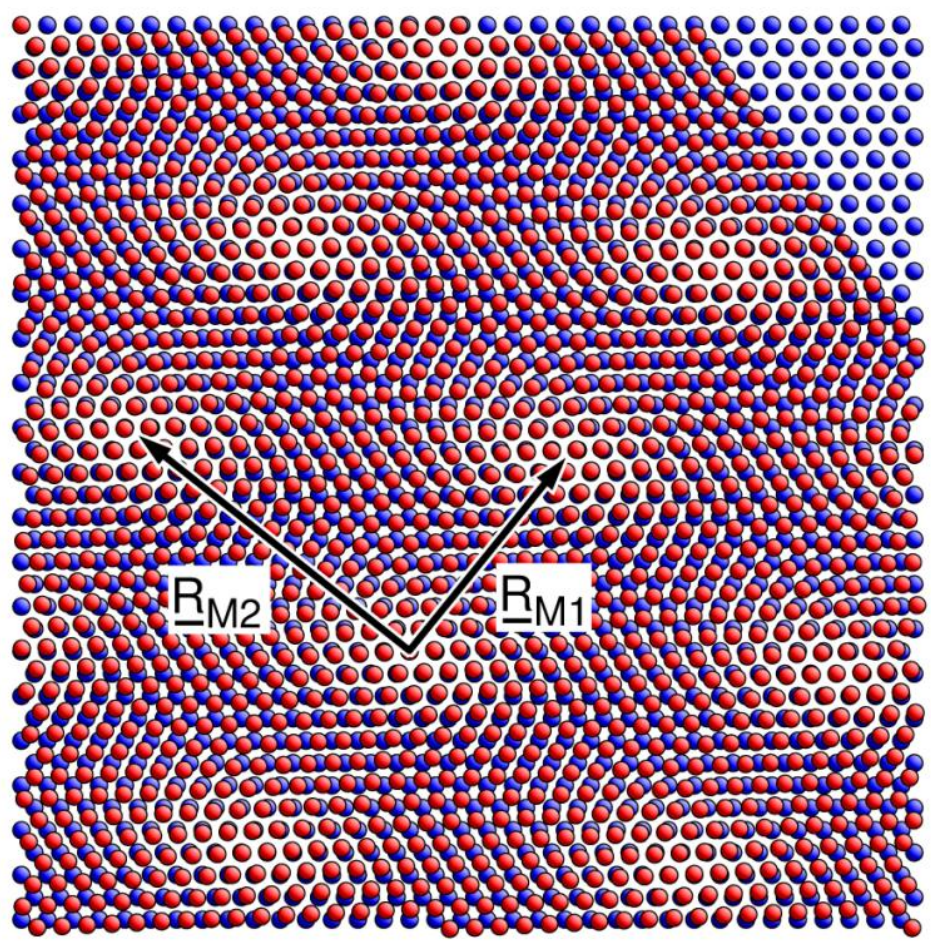

Fig. 12. Moiré pattern of a fictitious hexagonal overlayer on hexagonal substrate described as $(0.940 \times 0.983) \mathrm{R} 5^{\circ}$. Moiré vectors $\underline{\mathrm{R}}_{\mathrm{M} 1}, \underline{\mathrm{R}}_{\mathrm{M} 2}$ are shown by arrows and labeled accordingly. The periodicity of the substrate layer is illustrated by removing the overlayer at the upper right of the section. 